\title{
NONDENSITY OF THE ORBITAL SHADOWING PROPERTY IN $C^{1}$-TOPOLOGY
}

\author{
A. V. OSIPOV
}

\begin{abstract}
The orbital shadowing property (OSP) of discrete dynamical systems on smooth closed manifolds is considered. The nondensity of OSP with respect to the $C^{1}$-topology is proved. The proof uses the method of skew products developed by Ilyashenko and Gorodetskiı̌.
\end{abstract}

\section{§1. INTRODUCTION}

The theory of shadowing studies the closeness of approximate and exact trajectories (or orbits) of dynamical systems on unbounded time intervals. This problem is important both for applications (as a rule, approximate trajectories generated by computer simulation of a system are considered) and for the qualitative theory of dynamical systems (shadowing properties can be viewed as weak forms of structural stability). Note that we consider only discrete-time dynamical systems (cascades) generated by homeomorphisms of metric spaces and diffeomorphisms of closed smooth manifolds. In this paper, we do not distinguish between a homeomorphism and the dynamical system generated by this homeomorphism. Roughly speaking, a cascade has a shadowing property if any "sufficiently precise" approximate trajectory is "close" to an exact one. Since the statement that approximate trajectories (pseudotrajectories) and exact trajectories are close can be formalized in various ways, there are several shadowing properties. We mention the pseudo-orbit tracing property POTP, the orbital shadowing property OSP, and the weak shadowing property WSP. The state of the art in the theory of shadowing was described in the monographs [13, 11. The precise definitions of the shadowing properties that are used in this paper are as follows.

Let $f$ be a homeomorphism of a metric space $M$ with metric dist. We recall the definitions of the exact trajectory of a point $p \in M$ under $f$ and its positive and negative semitrajectories:

$$
\begin{aligned}
O(p, f) & =\left\{f^{k}(p) \mid k \in \mathbb{Z}\right\}, \\
O_{+}(p, f) & =\left\{f^{k}(p) \mid k \in \mathbb{Z}, k \geq 0\right\}, \\
O_{-}(p, f) & =\left\{f^{k}(p) \mid k \in \mathbb{Z}, k \leq 0\right\}
\end{aligned}
$$

(hereinafter, we denote by $\mathbb{Z}$ the set of integers).

For convenience, without special mention, we often use the notation

$$
p_{k}=f^{k}(p) \text { for } k \in \mathbb{Z} .
$$

Moreover, we sometimes identify a periodic point with its trajectory, i.e., the set $O(p, f)$.

2010 Mathematics Subject Classification. Primary 37C50.

Key words and phrases. Shadowing, generic property, skew product, $C^{1}$-topology. 
As usual, we say that a sequence $\xi=\left\{x_{k}\right\} \subset M$ is a $d$-pseudotrajectory if

$$
\operatorname{dist}\left(x_{k+1}, f\left(x_{k}\right)\right)<d \text { for } k \in \mathbb{Z} \text {. }
$$

Thus, a $d$-pseudotrajectory is a possible formalization of the notion of an approximate trajectory.

We say that a homeomorphism $f$ of the space $M$ has POTP (pseudo-orbit tracing property) if for any $\epsilon>0$ there exists $d$ such that for any $d$-pseudotrajectory $\xi=\left\{x_{k}\right\}$ there is a point $q \in M$ with

$$
\operatorname{dist}\left(x_{k}, f^{k}(q)\right)<\epsilon \text { for } k \in \mathbb{Z} .
$$

In other words, POTP means that any "sufficiently precise" approximate trajectory is shadowed by an exact trajectory (i.e., is pointwise close to it).

We denote by $N(\epsilon, A)$ the $\epsilon$-neighborhood of a set $A \subset M$. In the paper [12, definitions of the orbital shadowing property (OSP) and the weak shadowing property (WSP) were given. We say that a homeomorphism $f$ of the space $M$ has OSP (and write $f \in$ OSP) if for any $\epsilon>0$ there exists $d>0$ such that for any $d$-pseudotrajectory $\xi$ there is a point $q \in M$ such that

$$
\xi \subset N(\epsilon, O(q, f)) \quad \text { and } \quad O(q, f) \subset N(\epsilon, \xi) .
$$

A homeomorphism $f$ of $M$ is said to have WSP if for any $\epsilon>0$ there exists $d>0$ such that for any $d$-pseudotrajectory $\xi$ there is a point $q \in M$ with

$$
\xi \subset N(\epsilon, O(q, f)) \text {. }
$$

OSP is a weak analog of POTP: the difference is that we do not require a point $x_{k}$ of a pseudotrajectory $\xi=\left\{x_{k}\right\}$ and the point $f^{k}(q)$ of an exact trajectory $O(q, f)$ to be close "at any time moment"; instead, the sets of the points of $\xi$ and $O(q, f)$ are required to be close. The weak shadowing property WSP is a weak version of OSP: the difference is that a set of points of a "sufficiently precise" pseudotrajectory $\xi$ is required to be contained in a small neighborhood of some exact trajectory $O(q, f)$.

Let $M$ be a closed smooth manifold. As usual, we denote by $\operatorname{Diff}^{1}(M)$ the set of diffeomorphisms of $M$ with the $C^{1}$-topology (cf., e.g., 4 for the definition).

Generic properties are objects of special interest in the theory of dynamical systems. We say that a property is generic if it occurs for all cascades in a Baire second category set (cf., e.g., 4] for the definition) in a space of dynamical systems with a certain topology, and we say that a property is dense if it occurs for all cascades in a dense set. In the paper [15, Pilyugin and Plamenevskaya proved that POTP is generic with respect to $C^{0}$-topology if the phase space is a closed smooth manifold (hereinafter, we consider precisely this case). As long as POTP is $C^{0}$-generic, so are OSP and WSP. Bonatti, Diaz and Turcat 6] proved that POTP is nondense with respect to $C^{1}$-topology, and S. Crovisier 7 proved that WSP is $C^{1}$-generic (also, cf. the paper 14 by Pilyugin, Sakai, and Tarakanov).

Our main goal is to prove that OSP (which takes an "intermediate" position between WSP and POTP) is $C^{1}$-nondense (and, therefore, $C^{1}$-nongeneric). As usual, we denote by $S^{2}$ the two-dimensional sphere and by $S^{1}$ the circle. Our main result is the following theorem.

Theorem A. There exists a domain $W \subset \operatorname{Diff}^{1}\left(S^{2} \times S^{1}\right)$ such that any diffeomorphism $f \in W$ does not have OSP.

In order to prove this, we use an idea originating from the work of Gorodetskil and Ilyashenko: to look for an example in the class of partially hyperbolic skew products. Specifically, we consider a certain step skew product $G_{0}$ over the Bernoulli shift $\sigma$ with fiber homeomorphic to the circle (all necessary definitions will be given later). We realize 
the Bernoulli shift as a mapping of the Smale horseshoe in such a way that this map contracts and expands sufficiently fast compared to the fiber dynamics. Then we see that a local maximal partially hyperbolic set with center fiber homeomorphic to the circle corresponds to this skew product. Furthermore, the technique of Hirsch-Pugh-ShubGorodetskiǔ (cf. [4, 9, 8]) implies that the partially hyperbolic set persists under small perturbations of this smooth realization, still remaining the product of the circle and the Smale horseshoe. Next, due to the Hölder dependence of the (individually smooth) center fiber on the point in the base (i.e., in the Smale horseshoe), this skew product is a Hölder mild skew product.

We take a sufficiently small $C^{1}$-neighborhood of the step skew product $G_{0}$ for the role of the required neighborhood $W$ from Theorem A. In particular, this neighborhood is chosen so small that any diffeomorphism in $W$ is assigned to some mild skew product. Furthermore, we show that Theorem A can be reduced to Theorem A' (its precise formulation is given in the next section).

Theorem A'. Any Hölder mild skew product "sufficiently close" to the skew product $G_{0}$ does not have OSP.

The proof of Theorem A' is split into two cases. The first case (Case (A1)) corresponds to the situation where there exist two hyperbolic periodic points $p$ (with one-dimensional unstable manifold) and $q$ (with one-dimensional stable manifold) such that these manifolds intersect. In this case, using the Main Lemma, we construct a pseudotrajectory that cannot be orbitally shadowed by any exact trajectory.

The second case (Case (A2)) corresponds to the situation where there are no such intersections. In this case, we construct a pseudotrajectory such that any exact trajectory that orbitally shadows this pseudotrajectory should be a heteroclinic trajectory connecting two hyperbolic periodic points with one-dimensional unstable and one-dimensional stable manifold, respectively. The assumption that the cascade has OSP contradicts the assumption about the absence of such intersections.

We describe the further structure of the paper. In $\S 2$, the main definitions are given, the main properties of skew products are described, and it is shown that Theorem A can be reduced to Theorem $\mathrm{A}^{\prime}$. In $\S 3$, Lemma 1 (Main Lemma), which plays a significant role in the proof of Theorem $\mathrm{A}^{\prime}$, is formulated and proved. In $\S 4$, it is shown that the proof of Theorem $\mathrm{A}^{\prime}$ can be reduced to consideration of two cases: Case (A1) and Case (A2), and Case (A1) is analyzed. In addition, a draft of the proof of Theorem $\mathrm{A}^{\prime}$ is briefly outlined at the beginning of $\S 4$. In $\S 5$, two auxiliary lemmas on properties of the skew products under consideration, which are required for the proof of Case (A2), are formulated and verified. In $\S 6$, Case (A2) is analyzed, with the exception of Lemma 6. Lemma 6 is proved in $\S 7$, which consists of 4 subsections. In Subsection 7.1, the main notions required for the proof of Lemma 6 are introduced. In Subsection 7.2, outlines of proofs of Lemmas 8 and 9, playing a key role in the proof of item (6.c) of Lemma 6 , are given. In Subsection 7.3, the proof of item (6.c) is completed, and finally, in Subsection 7.4, the remaining items of Lemma 6 are proved.

\section{§2. Dynamical properties of SKeW products}

Our main definitions are as follows.

By $\Sigma^{2}$ we denote the space of all two-sided sequences of 0 's and 1's with the metric

$$
d_{\Sigma^{2}}\left(\omega, \omega^{\prime}\right)=1 / 2^{k}
$$

where $k \geq 0$ is the minimal integer such that, if

$$
\omega=\ldots \beta_{-1} \mid \beta_{0} \beta_{1} \ldots
$$


and

then

$$
\omega^{\prime}=\ldots \beta_{-1}^{\prime} \mid \beta_{0}^{\prime} \beta_{1}^{\prime} \ldots
$$

$$
\beta_{-k-1} \neq \beta_{-k-1}^{\prime} \quad \text { or } \quad \beta_{k} \neq \beta_{k}^{\prime},
$$

and the sign $\mid$ means that the next symbol stands at the zero position. The sign | will be used in what follows in the paper. Recall the definition of the Bernoulli shift $\sigma: \Sigma^{2} \mapsto \Sigma^{2}$ :

$$
\sigma\left(\ldots \beta_{-1} \mid \beta_{0} \beta_{1} \beta_{2} \ldots\right)=\ldots \beta_{-1} \beta_{0} \mid \beta_{1} \beta_{2} \ldots
$$

In the paper [1], the following definitions were introduced.

Definition 1. Fix two diffeomorphisms $f_{0}$ and $f_{1}$ of the circle $S^{1}$. A step skew product is a mapping $G: \Sigma^{2} \times S^{1} \mapsto \Sigma^{2} \times S^{1}$ such that

$$
G(\omega, \phi)=\left(\sigma(\omega), f_{\omega_{0}}(\phi)\right) \text { for } \omega \in \Sigma^{2}, \phi \in S^{1},
$$

where $\omega_{0}$ is the symbol standing at the zero position in the sequence $\omega$.

Definition 2. Fix a family of diffeomorphisms $f_{\omega}$ of the circle $S^{1}$ that is parametrized by two-sided sequences $\omega \in \Sigma^{2}$. A mild skew product is a mapping $G: \Sigma^{2} \times S^{1} \mapsto \Sigma^{2} \times S^{1}$ such that

$$
G(\omega, \phi)=\left(\sigma(\omega), f_{\omega}(\phi)\right) \quad \text { for } \omega \in \Sigma^{2}, \phi \in S^{1} .
$$

We emphasize that in Definition 1, the choice of the diffeomorphism $f_{j}$ is completely determined by the symbol $\omega_{0}$ standing at the zero position in the sequence $\omega$, whereas in Definition 2, the choice depends on the entire sequence $\omega$. We denote by $g$ a diffeomorphism of the sphere $S^{2}$ that has a standard Smale horseshoe. It is well known that the mapping $g$ has a locally maximal invariant subset $\Lambda$ homeomorphic to the set $\Sigma^{2}$, and that the restriction of $g$ to $\Lambda$ is topologically conjugate to the Bernoulli shift $\sigma$ (see, e.g., 10]).

It is well known (see, e.g., [1]) that the diffeomorphism $g: S^{2} \mapsto S^{2}$ can be viewed as a mapping $g: D_{0} \cup D_{1} \mapsto D_{0}^{\prime} \cup D_{1}^{\prime}$, where $D_{0}$ and $D_{1}$ are disjoint horizontal rectangles, and $D_{0}^{\prime}$ and $D_{1}^{\prime}$ are disjoint vertical rectangles. In the following definition (which is also taken from [1]), we extend a step skew product to the set $\left(D_{0} \cup D_{1}\right) \times S^{1}$ :

Definition 3. A smooth realization of a step skew product $G$ is a smooth mapping $F:\left(D_{0} \cup D_{1}\right) \times S^{1} \mapsto\left(D_{0}^{\prime} \cup D_{1}^{\prime}\right) \times S^{1}$ such that

$$
\begin{gathered}
F(x, \phi)=\left(g(x), f_{x}(\phi)\right) \text { for } x \in D_{0} \cup D_{1}, \phi \in S^{1}, \\
\text { where } f_{x}=f_{j} \text { for } x \in D_{j}, j \in\{0,1\},
\end{gathered}
$$

and $f_{0}$ and $f_{1}$ are the diffeomorphisms occurring in the definition of the step skew product $G$.

The smooth realization $F$ of a skew product $G$ can be extended smoothly to a diffeomorphism of the manifold $M=S^{2} \times S^{1}$. We denote this extension by $F$ again, and, hereinafter, by a smooth realization we mean precisely a diffeomorphism of the manifold $M$. It is easily seen that the diffeomorphism $F$ has a locally maximal invariant set such that the dynamics on this set coincides with the dynamics of the initial skew product $G$.

Let $g_{0}$ be the rotation of the circle $S^{1}$ by a small angle $b<1 / 100$. Let $g_{1}$ be an orientation-preserving diffeomorphism whose nonwandering set consists only of two fixed points: an attractor $p$ and a repeller $q$. As usual, we regard $S^{1}$ as $\mathbb{R} / \mathbb{Z}$. We assume that the mapping $g_{1}$ is a linear expansion with some constant $a>1$ in the neighborhood of the point $q=0$ of radius $1 / 8$ and the linear contraction with the constant $1 / a$ in the 
neighborhood of the point $p=1 / 2$ of radius $1 / 8$. As usual, we denote by id the identity map. In addition, we assume that the diffeomorphisms $g_{0}$ and $g_{1}$ satisfy the formula

$$
\operatorname{dist}_{C^{1}}\left(g_{j}, \text { id }\right)<\delta_{0} \quad \text { for } j \in\{0,1\} \text {, }
$$

where $\delta_{0}$ is a sufficiently small number (we shall impose restrictions on the size of $\delta_{0}$ below, in Theorem 2). Let $G_{0}$ denote the step skew product generated by the diffeomorphisms $g_{0}$ and $g_{1} . G_{0}$ is precisely the skew product discussed in $\S 1$.

The set $\Sigma^{2}$ is called the base, and any set of the form $\omega \times S^{1}$, where $\omega \in \Sigma^{2}$, is called a fiber. We denote by $p r: \Sigma^{2} \times S^{1} \mapsto \Sigma^{2}$ the natural projection onto the base. We say that the trajectories of points $p_{1}, p_{2} \in \Sigma^{2} \times S^{1}$ lie on different fibers if the trajectories of their projections to the base do not intersect. Any finite sequence of 0's or 1's is called a word.

A definition of a hyperbolic periodic point was given in the book [5]. Let $p$ be a hyperbolic periodic point of a diffeomorphism $f$ of a manifold $M$. We define the sets

$$
\begin{aligned}
& W^{s}(p)=\left\{q \in M \mid \exists r \in O(p, f): \operatorname{dist}\left(f^{k}(q), f^{k}(r)\right) \longrightarrow 0, k \rightarrow+\infty\right\}, \\
& W^{u}(p)=\left\{q \in M \mid \exists r \in O(p, f): \operatorname{dist}\left(f^{k}(q), f^{k}(r)\right) \longrightarrow 0, k \rightarrow-\infty\right\} .
\end{aligned}
$$

For convenience, we call the sets defined by (2.1) and (2.2) the stable and the unstable manifold of $p$, respectively. Note that usually these sets are called the stable and unstable manifolds of the trajectory $O(p, f)$. We say that $p$ is a point of type $(m, n)$ if

$$
\operatorname{dim} W^{s}(p)=m \quad \text { and } \quad \operatorname{dim} W^{u}(p)=n .
$$

A periodic point $p \in \Sigma^{2}$ of the Bernoulli shift $\sigma$ is called a hyperbolic periodic point of type $(1,1)$. In accordance with this definition, a hyperbolic periodic point of type $(1,1)$ of the diffeomorphism $g: S^{2} \mapsto S^{2}$ corresponds to any hyperbolic periodic point $p \in \Sigma^{2}$. A periodic point $p=\left(\omega_{0}, \phi_{0}\right) \in \Sigma^{2} \times S^{1}$ is called a hyperbolic periodic point of type $(2,1)$ (of type $(1,2)$, respectively) of a mild skew product $G$ if it is a hyperbolic attracting (repelling) point of the diffeomorphism

$$
\bar{G}_{\omega_{0}, \phi_{0}}: S^{1} \mapsto S^{1}, \quad \bar{G}_{\omega_{0}, \phi_{0}}(\phi):=\operatorname{pr}_{S^{1}} G^{m_{p}}\left(\omega_{0}, \phi\right) \text { for } \phi \in S^{1},
$$

where $\operatorname{pr}_{S^{1}}$ is a projection onto $S^{1}$, and $m_{p}$ is the period of the point $p$.

We use a result of Ilyashenko and Gorodetskil on the density of hyperbolic periodic points of different types; in fact, this result is a consequence of Theorem 2 in [2].

Theorem 1 (Gorodetskiı̌, Ilyashenko). For the diffeomorphisms $g_{0}$ and $g_{1}$ defined above and any numbers $C$ and $\alpha$, there exist neighborhoods $W_{0}\left(g_{0}\right)$ and $W_{1}\left(g_{1}\right)$ (in $C^{1}$-topology) such that if a mild skew product $G$ (generated by the diffeomorphisms $f_{\omega}$ ) satisfies the conditions

$$
f_{\omega} \in W_{\omega_{0}} \quad \text { for } \omega \in \Sigma^{2}
$$

(where $\omega_{0}$ is the symbol standing at the zero position of the sequence $\omega$ ),

$$
L:=\max _{\omega \in \Sigma^{2}} \max _{\phi \in S^{1}}\left(\left\|D f_{\omega}(\phi)\right\|,\left\|D f_{\omega}^{-1}(\phi)\right\|\right)<2^{\alpha},
$$

and

$$
d_{C^{0}}\left(f_{\omega}, f_{\omega^{\prime}}\right) \leq C\left(d_{\Sigma^{2}}\left(\omega, \omega^{\prime}\right)\right)^{\alpha} \quad \text { for } \omega, \omega^{\prime} \in \Sigma^{2},
$$

where $d_{C^{0}}$ is the $C^{0}$-metric, then the hyperbolic periodic points of type $(2,1)$, as well as the hyperbolic periodic points of type $(1,2)$, are dense in the set $\Sigma^{2} \times S^{1}$.

Note that if the diffeomorphisms $f_{\omega}$ satisfy (2.5), then the mild skew product $G$ is called a Hölder mild skew product. In fact, Theorem 1 states that the hyperbolic periodic points of different types are dense for Hölder mild skew products "sufficiently close" to the skew product $G_{0}$. 
Let $F_{0}$ denote the smooth realization of the step skew product $G_{0}$. The following theorem plays an important role in the proof of Theorem A. In fact, the theorem states that any diffeomorphism close to the diffeomorphism $F_{0}$ has a local maximal invariant set such that the dynamics on this set coincides with the dynamics of some Hölder skew product "close" to the skew product $G_{0}$.

Theorem 2. Suppose that the diffeomorphisms $g_{0}$ and $g_{1}$ defined above are sufficiently $C^{1}$-close to the identity diffeomorphism (i.e., the number $\delta_{0}$ defined above is sufficiently small). Then there exist numbers $C, \alpha$ and a neighborhood $W$ of the diffeomorphism $F_{0}$ in $C^{1}$-topology such that any diffeomorphism $F \in W$ has a local maximal invariant set $\Delta$, and $\left.F\right|_{\Delta}$ is topologically conjugate to a mild skew product $G$ that satisfies all conditions of Theorem 1 (namely, conditions (2.3), (2.4), and (2.5)).

Remark 1. Apparently, Theorem 2 has not been formulated explicitly anywhere, but basically, its proof was given in the papers [3, 4, 8. In Theorem 2, a (nonunique) mild skew product $G$ is assigned to a diffeomorphism $F$ sufficiently close to the identity. In the papers [3, 4, 8, it was proved that this correspondence is continuous. Note that the arguments in the papers 4, 8] imply that the hyperbolic periodic points that belong to the sets $\Delta$ related to diffeomorphisms in $W$ are assigned to hyperbolic periodic points of the same type of mild skew products.

In order to prove the main result, we need the following notation.

For convenience, we denote by dist both the metric on the manifold $S^{2} \times S^{1}$ and the metric in the space $\Sigma^{2} \times S^{1}$. Let $p$ be a hyperbolic periodic point of a homeomorphism $f$ of a metric space $M$. The set defined by (2.1) (by (2.2), respectively) is called the stable (respectively, unstable) manifold of $p$. Choose a mild skew product $G$ from Theorem 2 as a homeomorphism $f$. Let $F$ be the diffeomorphism of the manifold $M$ that corresponds to the mild skew product $G$. We emphasize that the sets $W^{s}(p)$ and $W^{u}(p)$ are not manifolds, in spite of their names. However, the sets $W_{F}^{s}(\bar{p})$ and $W_{F}^{u}(\bar{p})$, i.e., the stable and the unstable manifold of the trajectory of the point $\bar{p} \in S^{2} \times S^{1}$ (which corresponds to the point $p \in \Sigma^{2} \times S^{1}$ in the sense of Theorem 2) with respect to the diffeomorphism $F$, are manifolds. We define the dimensions of the sets $W^{s}(p)$ and $W^{u}(p)$ by the formulas

$$
\operatorname{dim} W^{s}(p):=\operatorname{dim} W_{F}^{s}(\bar{p}), \quad \operatorname{dim} W^{u}(p):=\operatorname{dim} W_{F}^{u}(\bar{p}) .
$$

Thus, if $p$ is a hyperbolic periodic point of type $(m, n)$, then $\operatorname{dim} W^{s}(p)=m$ and $\operatorname{dim} W^{u}(p)=n$.

If $p$ is a periodic point of the Bernoulli shift $\sigma$, then the numbers $\operatorname{dim} W^{s}(p)$ and $\operatorname{dim} W^{u}(p)$ can be defined in a similar way with the exception that a diffeomorphism $F$ should be replaced by the diffeomorphism $g$ defined above. In accordance with this definition, $\operatorname{dim} W^{s}(p)=\operatorname{dim} W^{u}(p)=1$; i.e., the point $p \in \Sigma^{2}$ is a hyperbolic periodic point of type $(1,1)$.

Let $W$ be the neighborhood of the mapping $F_{0}$ from Theorem 2 . In order to prove Theorem 2, it suffices to find a number $\delta^{\prime}$ such that $N\left(\delta^{\prime}, F_{0}\right) \subset W$, and any diffeomorphism $F \in N\left(\delta^{\prime}, F_{0}\right)$ does not have OSP. Let $\delta$ be an arbitrary number. By Remark 1, if $\delta^{\prime}$ is sufficiently small, then any diffeomorphism $F \in N\left(\delta^{\prime}, F_{0}\right)$ is assigned to some mild skew product $G$, and the diffeomorphisms $f_{\omega}$ of the mild skew product $G$ (see Definition 2) are contained in the neighborhood $N\left(\delta, g_{\omega_{0}}\right)$, where the symbol $\omega_{0}$ has the same meaning as in Theorem 1. In the course of the proof of Theorem A, we fix a sufficiently small number $\delta$; i.e., we also fix the number $\delta^{\prime}$, implicitly. By Theorem 2 , any diffeomorphism $F \in W$ has the local maximal invariant set $\Delta$. Hence, in order to prove Theorem A, it suffices to establish only that the restriction $\left.F\right|_{\Delta}$ does not have OSP. Since OSP is preserved under conjugacy, Theorem A reduces to the following theorem. 
Theorem $\mathbf{A}^{\prime}$. Let $G$ be a mild skew product corresponding (in the sense of Remark 1) to some diffeomorphism $F$ of the manifold $M=S^{2} \times S^{1}$. Also, suppose that the skew product $G$ satisfies the conditions of Theorem 1 and that the neighborhoods $W_{0}\left(g_{0}\right)$ and $W_{1}\left(g_{1}\right)$ are sufficiently small. Then $G$ does not have OSP.

\section{§3. MAIN Lemma}

The following lemma is a main ingredient of our constructions. It gives a sufficient condition for a pseudotrajectory to satisfy the following: any exact trajectory that orbitally shadows this pseudotrajectory is contained in the stable (or the unstable) manifold of a hyperbolic fixed point.

Lemma 1. Let $M$ be a closed smooth manifold with metric dist, $f$ a diffeomorphism of the manifold $M, p$ a hyperbolic periodic point, and let $q^{1} \in W^{s}(p)$. Fix numbers $R>0$ and $0<\epsilon_{0}<R / 2$. There exists a number $0<\epsilon<\epsilon_{0}$ such that if a sequence $\xi=\left\{x_{k}\right\}$ satisfies

$$
x_{k}=q_{k}^{1}=f^{k}\left(q^{1}\right) \quad \text { for } k \geq 1, \quad x_{k} \notin N\left(\epsilon_{0}, O(p, f)\right) \quad \text { for } k<1,
$$

then for any point $q^{2}$ that satisfies the inclusions

$$
\xi \subset N\left(\epsilon, O\left(q^{2}, f\right)\right) \text { and } O\left(q^{2}, f\right) \subset N(\epsilon, \xi)
$$

and the inequality $\operatorname{dist}\left(q_{1}^{1}, q_{1}^{2}\right)<\epsilon$, we have

$$
\begin{aligned}
q^{2} & \in W^{s}(p), \\
\operatorname{dist}\left(q_{k}^{1}, q_{k}^{2}\right) & \leq R \quad \text { for } k \geq 1 .
\end{aligned}
$$

In fact, the lemma states the following. Let $p$ be a hyperbolic periodic point. Then, if a pseudotrajectory $\xi=\left\{x_{k}\right\}$ of a certain type is "close" to the trajectory $O(p, f)$ for all sufficiently large positive $k$ and is "far" from the trajectory $O(p, f)$ for all negative $k$ with sufficiently large absolute values, then any exact trajectory that orbitally shadows the pseudotrajectory $\xi$ is contained in the stable manifold of the point $p$.

Corollary. Under the conditions of Lemma 1 , suppose that $q^{1} \in W^{u}(p)$. There exists a number $0<\epsilon<\epsilon_{0}$ such that if a sequence $\xi=\left\{x_{k}\right\}$ is such that

$$
x_{k}=q_{k}^{1}=f^{k}\left(q^{1}\right) \quad \text { for } k \leq 1, \quad x_{k} \notin N\left(\epsilon_{0}, O(p, f)\right) \quad \text { for } k>1,
$$

then for any point $q^{2}$ that satisfies the inequality $\operatorname{dist}\left(q_{1}^{1}, q_{1}^{2}\right)<\epsilon$ and the inclusions (3.2), the following is true:

$$
\begin{gathered}
q^{2} \in W^{u}(p), \\
\operatorname{dist}\left(q_{k}^{1}, q_{k}^{2}\right) \leq R \quad \text { for } \quad k \leq 1 .
\end{gathered}
$$

Proof of Lemma 1. First, we need to choose a sufficiently small $\epsilon$. We do this in several steps.

Choose a number $\epsilon_{1}<\epsilon_{0}$ such that

- if $O(p, f)=\left\{p_{0}, \ldots, p_{m_{p}-1}\right\}$, then the neighborhoods

$$
N\left(\epsilon_{1}, p_{0}\right), N\left(\epsilon_{1}, p_{1}\right), \ldots, N\left(\epsilon_{1}, p_{m_{p}-1}\right)
$$

are disjoint;

- if a positive semitrajectory $O_{+}(x, f)$ is contained in the set $N\left(\epsilon_{1}, O(p, f)\right)$, then $x \in W^{s}(p)$

- $\left(N\left(\epsilon_{1}, O(p, f)\right) \cup f\left(N\left(\epsilon_{1}, O(p, f)\right)\right)\right) \cap N\left(\epsilon_{1}, x_{k}\right)=\varnothing$ for $k \leq 1$;

- there are no points of the sequence $\xi$ on the boundary of the set $N\left(\epsilon_{1}, O(p, f)\right)$. 
Let $n>1$ be the minimal number such that the points $x_{k}$ are contained in the neighborhood $N\left(\epsilon_{1}, O(p, f)\right)$ for $k \geq n$. Choose a number $\epsilon_{2}<\epsilon_{1}$ such that

- the following neighborhoods are disjoint:

$$
N\left(\epsilon_{2}, O(p, f)\right) \text { and } N\left(\epsilon_{2}, x_{k}\right) \text { for } 1 \leq k \leq n ;
$$

- if there exist numbers $1 \leq k_{1} \leq n$ and $1 \leq k_{2}$ such that

$$
x_{k_{1}}=q_{k_{1}}^{1} \notin N\left(\epsilon_{1}, O(p, f)\right) \quad \text { and } \quad x_{k_{2}}=q_{k_{2}}^{1} \in N\left(\epsilon_{1}, O(p, f)\right) \text {, }
$$

then $N\left(\epsilon_{1}, O(p, f)\right) \cap N\left(\epsilon_{2}, x_{k_{1}}\right)=\varnothing$ and $N\left(\epsilon_{2}, x_{k_{2}}\right) \subset N\left(\epsilon_{1}, O(p, f)\right)$.

Let $m \geq n$ be the minimal number such that the point $x_{m}$ is contained in the set $N\left(\epsilon_{2} / 3, O(p, f)\right)$. Choose a number $\epsilon_{3}<\epsilon_{2} / 3$ such that the neighborhoods $N\left(\epsilon_{3}, x_{k}\right)$ are disjoint for $1 \leq k \leq m$. Finally, choose a number $\epsilon<\epsilon_{3}$ such that

$$
f^{j-k}\left(N\left(\epsilon, x_{k}\right)\right) \subset N\left(\epsilon_{3}, x_{j}\right) \text { for } j, k \in\{1, \ldots, m\} .
$$

Now we show that the number $\epsilon$ chosen above has the desired properties. Suppose that relation (3.2) holds true for a point $q^{2}$ and for the sequence $\xi$.

Since $q_{1}^{2} \in N\left(\epsilon, x_{1}\right)$, the choice of $\epsilon$ implies that

$$
q_{k}^{2} \in N\left(\epsilon_{3}, x_{k}\right) \text { for } 1 \leq k \leq m .
$$

Thus, by (3.8) and our notation, the point $q_{2}^{m}$ is contained in the set $N\left(\epsilon_{2}, O(p, f)\right)$. In order to obtain (3.3), it suffices to prove that

$$
q_{k}^{2} \in N\left(\epsilon_{1}, O(p, f)\right) \quad \text { for } k \geq m .
$$

Suppose that (3.9) fails, i.e., that there exists a minimal number $r>m$ such that

$$
q_{r}^{2} \notin N\left(\epsilon_{1}, O(p, f)\right) .
$$

From relation (3.2) and the choice of $\epsilon_{2}$ and $\epsilon_{3}$, it follows that there exists a number $k<n$ such that the point $q_{r}^{2}$ is contained in the set $N\left(\epsilon, x_{k}\right)$. Two cases are possible:

Case 1: $k \leq 1$. By the choice of $r$, we have $q_{r-1}^{2} \in N\left(\epsilon_{1}, O(p, f)\right)$. But then, by (3.10),

$$
f\left(N\left(\epsilon_{1}, O(p, f)\right)\right) \cap N\left(\epsilon_{1}, x_{k}\right) \neq \varnothing \quad \text { for } k \leq 1,
$$

which contradicts the choice of $\epsilon$.

Case 2: $1<k<n$. There exists a maximal number $1 \leq k^{\prime}<k$ such that $x_{k^{\prime}}$ does not belong to the set $N\left(\epsilon_{1}, O(p, f)\right)$. But then, by the choice of $\epsilon$,

$$
q_{r-t}^{2} \in N\left(\epsilon_{3}, x_{k-t}\right) \quad \text { for } 0 \leq t \leq k-k^{\prime},
$$

and hence, by the choice of $\epsilon_{2}$, we have

$$
q_{r-\left(k-k^{\prime}\right)}^{2} \notin N\left(\epsilon_{1}, O(p, f)\right) \quad \text { and } \quad q_{r-t}^{2} \notin N\left(\epsilon_{2}, O(p, f)\right) \quad \text { for } 0 \leq t \leq k-k^{\prime} .
$$

Thus, $r-\left(k-k^{\prime}\right)>m$ (because otherwise, one could find a number $1 \leq t \leq k-k^{\prime}$ such that $q_{m}^{2}=q_{r-t}^{2}$ belongs to $\left.N\left(\epsilon_{2}, O(p, f)\right)\right)$. Hence, the number $r$ is not minimal, and we get the desired contradiction.

We have proved (3.9) and, with it, also (3.3). By (3.8) and (3.9), and since $q_{k}^{1} \in$ $N\left(\epsilon_{1}, O(p, f)\right)$ for $k \geq m$, inequality (3.4) holds true for $q^{1}$ and $q^{2}$.

Lemma 1 is proved. 


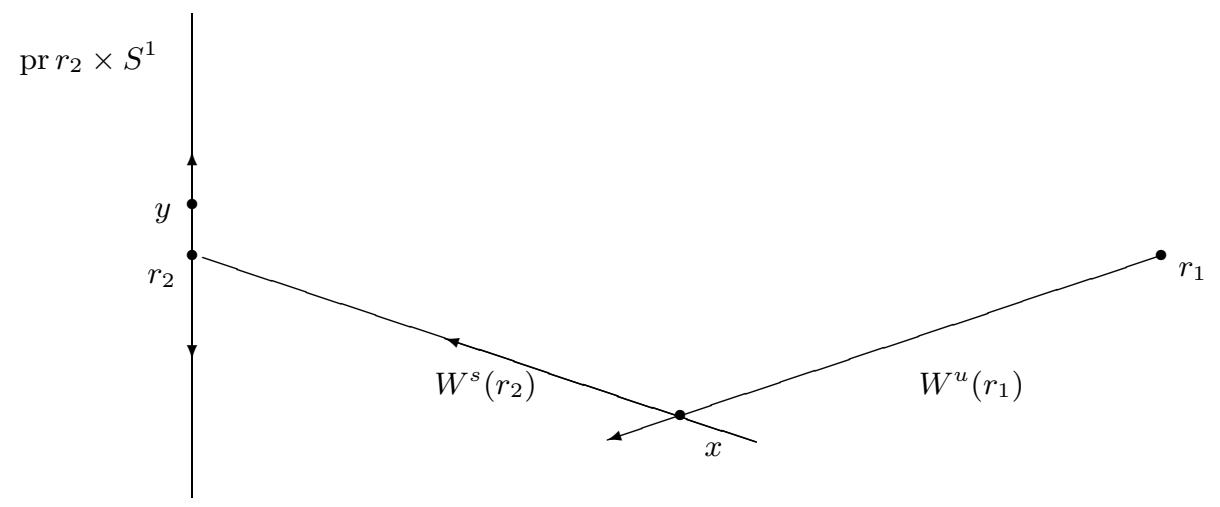

FiguRE 1

\section{§4. Reduction of the proof of Theorem A' to two CASes, AND THE PROOF IN CASE (A1)}

The following two cases are possible:

(A1) There exist two hyperbolic periodic points $r_{1}$ and $r_{2}$ lying on different fibers and with one-dimensional unstable and stable manifolds, respectively, such that these manifolds intersect.

(A2) For any two hyperbolic periodic points $r_{1}$ and $r_{2}$ lying on different fibers and with one-dimensional unstable and stable manifolds, respectively, these manifolds do not intersect.

Now we can give a more detailed outline of the method of proof in the two cases under consideration.

In Case (A1), we construct the pseudotrajectory in the following way. It includes a part of an exact heteroclinic trajectory from $r_{1}$ to $r_{2}$; then it "leaps" (sufficiently close to $r_{2}$ ) to a trajectory that lies on the fiber of $r_{2}$ and "goes away" from $r_{2}$ by its unstable manifold. We emphasize that the Main Lemma can be applied not only to the pseudotrajectory, but also to its projection to the base (the Smale horseshoe). To get a contradiction, suppose that the pseudotrajectory constructed is orbitally shadowed by an exact trajectory. Next, we apply the Main Lemma to the projection of the trajectory to the base and see that this trajectory lies on the fiber of some trajectory "going to" the point $r_{2}$. On the other hand, since the pseudotrajectory "outcomes from" $r_{1}$, the exact trajectory must be contained in the unstable manifold of the point $r_{1}$. Moreover, since the Bernoulli shift is expansive, the projection to the base of the heteroclinic trajectory used in our construction must coincide with the projection of the exact shadowing trajectory. Finally, since the projection of a local unstable manifold is one-to-one in a small neighborhood of $r_{1}$, and we know the point in the base, the exact trajectory must precisely coincide with the heteroclinic trajectory used in our construction. We arrive at a contradiction: the "final" part of the pseudotrajectory (near the point $r_{2}$ ) is not shadowed. The phase portrait of a mild skew product in Case (A1) is depicted in Figure 1.

In Case (A2), using the technique of mild skew products, we construct a pseudotrajectory that "outcomes from" and "comes to" hyperbolic periodic points with onedimensional unstable and stable manifolds in the initial and final phases, respectively, and does not approach these points in the intermediate phase. Then, by the Main Lemma, if an exact trajectory orbitally shadows the pseudotrajectory, then it must be a heteroclinic trajectory connecting these points, and we get the desired contradiction.

Case (A1) is proved in this section, and Case (A2) is considered in the remaining sections. 
Suppose that a mild skew product $G$ satisfies the conditions of Case (A1). Choose points $x \in W^{u}\left(r_{1}\right) \cap W^{s}\left(r_{2}\right)$ and $y \in\left(\operatorname{pr} r_{2} \times S^{1}\right) \cap W^{u}\left(r_{2}\right)$ (cf. Figure 1). First, we need to choose a sufficiently small number $\varepsilon$. We do it in several steps.

Choose a number $\varepsilon_{0}>0$ such that

1) the claim of the corollary to the Main Lemma holds true for the mapping $G$, some point of the trajectory $O(x, G)$, and the point $r_{1}$;

2) the claim of the Main Lemma holds true for the Bernoulli shift $\sigma$, some point of the trajectory $O(\operatorname{pr} x, \sigma)$, and the point $\operatorname{pr} r_{2}$ (in these two items the number $R$ is assumed to be sufficiently small, but fixed beforehand);

3 ) the point $y$ does not belong to $N\left(2 \varepsilon_{0}, O(x, G)\right)$;

4) the restriction of the projection pr to the local unstable manifold $W_{\varepsilon_{0}}^{u}\left(r_{1}\right)$ is one-to-one.

We comment on items 1) and 2). Strictly speaking, neither the Main Lemma nor its corollary can be applied directly in our case, because $\Sigma^{2}$ and $\Sigma^{2} \times S^{1}$ are not manifolds, while the statements mentioned above were proved only for manifolds. However, in item 1), we can consider the diffeomorphism $F$ of the manifold $M$ that corresponds to the mild skew product $G$ (in the sense of Remark 1), and points $\bar{x}$ and $\bar{r}_{1}$ that are analogs of the points $x$ and $r_{1}$ for $F$. There exists a number $\epsilon_{0}$ and a point $x_{0} \in O(x, G)$ that satisfy an analog of condition (3.5). Choose a point $q^{2} \in \Sigma^{2} \times S^{1}$ that satisfies an analog of relation (3.2) for some small $\epsilon$ and also satisfies the inequality $\operatorname{dist}\left(G\left(q^{2}\right), G\left(x_{0}\right)\right)<\epsilon$. By Theorem 2, there exists a homeomorphism $h_{F}$ conjugating the restriction $\left.F\right|_{\Delta}$ (where the set $\Delta$ is as defined in Theorem 2) and the mild skew product $G$. Since $h_{F}$ and $h_{F}^{-1}$ are homeomorphisms of compact metric spaces, they are uniformly continuous. Hence, an analog of condition (3.5) is fulfilled for some number $\bar{\epsilon}_{0}$ and point $\bar{x}_{0}=h_{F}^{-1}\left(x_{0}\right)$. Moreover, all conditions of the corollary are satisfied for the point $\bar{q}^{2}=h_{F}^{-1}\left(q^{2}\right)$ (an analog of the point $q^{2}$ ) and for the point $\bar{x}_{0}$, a sufficiently small number $\bar{\epsilon}$, and the mapping $F$. Thus, the corollary can be applied to the diffeomorphism $F$; i.e., analogs of relations (3.6) and (3.7) hold true for sufficiently small numbers $\bar{R}$ and $\bar{\epsilon}$, and the points $\bar{x}_{0}$ and $\bar{q}^{2}$. Hence, by the uniform continuity of the homeomorphism $h_{F}$, analogs of relations (3.6) and (3.7) hold true for sufficiently small numbers $R$ and $\epsilon$, and the points $x_{0}$ and $q^{2}$

Therefore, the claim of the corollary is valid for the mapping $G$. In item 2), we can consider the diffeomorphism $g: S^{2} \mapsto S^{2}$ (which was fixed above) having the Smale horseshoe, and apply a similar argument. Thus, the statement of Lemma 1 can also be applied to the Bernoulli shift $\sigma$.

We comment on item 4). Consider the diffeomorphism $F: M \mapsto M$ corresponding to the mild skew product $G$. By Theorem 2, the diffeomorphism $F$ has the local maximal invariant set $\Delta$ homeomorphic to $\Sigma^{2} \times S^{1}$ and such that $\left.F\right|_{\Delta}$ and $G$ are topologically conjugate. The sets homeomorphic to $S^{1}$ and corresponding to the fibers of the mild skew product $G$ are called the central fibers. By Remark 1, any periodic point $r_{1}$ of the mild skew product $G$ is assigned to some hyperbolic periodic point $\bar{r}_{1}$ of the diffeomorphism $F$. Since $r_{1}$ is a point of type $(2,1)$, the local unstable manifold of the point $\bar{r}_{1}$ with respect to the diffeomorphism $F$, i.e., the set $W_{\mathrm{loc}, F}^{u}\left(\bar{r}_{1}\right)$, is a finite union of "intervals". The angle between the unstable space of the diffeomorphism $F$ at the point $\bar{r}_{1}$ and the corresponding central fiber is not equal to zero. In the papers [3, 4, 9, it was shown that the central fibers of the diffeomorphism $F$ are $C^{1}$-close to the corresponding fibers of the diffeomorphism $g \times \operatorname{id}_{S^{1}}$, i.e., to circles. Hence, the intersection of the set $W_{\text {loc }, F}^{u}\left(\bar{r}_{1}\right)$ and any central fiber consists of at most one point. Consequently, the intersection of the set $W_{\mathrm{loc}, G}^{u}\left(r_{1}\right)$ and any fiber also consists of at most one point. Thus, the projection to the base is a one-to-one mapping on the set $W_{\mathrm{loc}, G}^{u}\left(r_{1}\right)$. 
We need the following two lemmas.

Lemma 2. There exists a number $R$ such that if $\sigma: \Sigma^{2} \mapsto \Sigma^{2}$ is the Bernoulli shift, and points $q^{1}, q^{2} \in \Sigma^{2}$ satisfy the inequality

$$
d_{\Sigma^{2}}\left(q_{k}^{1}, q_{k}^{2}\right) \leq R \text { for } k \in \mathbb{Z}
$$

then $q^{1}=q^{2}$ (recall that $\left.q_{k}^{j}=\sigma^{k}\left(q^{j}\right)\right)$.

In fact, Lemma 2 means that the Bernoulli shift is expansive. The proof of this fact is given, e.g., in the book [10].

Lemma 3. Under the conditions of Case (A1), let $R$ be the number given by Lemma 2 . There exists an $\varepsilon<\varepsilon_{0}$ such that if the relations

$$
\begin{gathered}
d_{\Sigma^{2}}\left(q_{1}^{1}, q_{1}^{2}\right)<\epsilon, \\
O\left(q^{1}, \sigma\right) \subset N\left(\varepsilon, O\left(q^{2}, \sigma\right)\right), \quad \text { and } O\left(q^{2}, \sigma\right) \subset N\left(\varepsilon, O\left(q^{1}, \sigma\right)\right)
\end{gathered}
$$

hold true for two points $q^{1}=\operatorname{pr} x$ and $q^{2}$ in $\Sigma^{2}$ such that $q^{1}, q^{2} \in W^{u}\left(\operatorname{pr} r_{1}\right) \cap W^{s}\left(\operatorname{pr} r_{2}\right)$, then $\operatorname{dist}\left(q_{k}^{1}, q_{k}^{2}\right) \leq R$ for $k \in \mathbb{Z}$.

In other words, Lemma 3 means that, under the conditions of Case (A1), if two heteroclinic trajectories which "go from" $\operatorname{pr} r_{1}$ "to" pr $r_{2}$ are orbitally close, then they are pointwise close.

Proof. By the choice of $\varepsilon_{0}$, the claim of the corollary to Lemma 1 is valid for the point $\operatorname{pr} r_{1}$, the mapping $\sigma$, and some point $q_{k_{1}-1}^{1}=\sigma^{k_{1}-1}\left(q^{1}\right) \in O(\operatorname{pr} x, \sigma)$, and the claim of Lemma 1 is valid for the point pr $r_{2}$, the mapping $\sigma$, and some point $q_{k_{2}-1}^{2} \in O(\operatorname{pr} x, \sigma)$ (with $R$ given by Lemma 2). Hence, if $d_{\Sigma^{2}}\left(q_{k_{1}}^{1}, q_{k_{1}}^{2}\right)<\varepsilon_{0}$, then, by inequalities (3.4),

$$
d_{\Sigma^{2}}\left(q_{k}^{1}, q_{k}^{2}\right) \leq R \text { for } k \leq k_{1} .
$$

Similarly, if $d_{\Sigma^{2}}\left(q_{k_{2}}^{1}, q_{k_{2}}^{2}\right)<\varepsilon_{0}$, then

$$
d_{\Sigma^{2}}\left(q_{k}^{1}, q_{k}^{2}\right) \leq R \text { for } k \geq k_{2} .
$$

Choose a number $\varepsilon<\varepsilon_{0}$ such that the inequalities $d_{\Sigma^{2}}\left(q_{1}^{1}, q_{1}^{2}\right)<\varepsilon$ imply the inequalities

$$
\begin{array}{lll}
d_{\Sigma^{2}}\left(q_{k}^{1}, q_{k}^{2}\right)<\varepsilon_{0} & \text { for } k \text { between } 1 \text { and } k_{1}, \\
d_{\Sigma^{2}}\left(q_{k}^{1}, q_{k}^{2}\right)<\varepsilon_{0} & \text { for } k \text { between } 1 \text { and } k_{2} .
\end{array}
$$

The number $\varepsilon$ has the desired properties; i.e., the claim of Lemma 3 holds true for this number.

Let $\varepsilon$ be the number whose existence was proved in Lemma 3. Choose an arbitrary number $d<\varepsilon$. Now we construct the pseudotrajectory discussed at the beginning of $\S 4$. Choose numbers $k_{1}$ and $k_{2}$ such that

$$
x_{k_{1}+1} \in N\left(d / 2, O\left(r_{2}, G\right)\right) \text { and } y_{k_{2}} \in N\left(d / 2, O\left(r_{2}, G\right)\right) .
$$

We construct the $d$-pseudotrajectory $\xi=\left\{\xi_{k}\right\}$ in the following way (cf. Figure 1):

$$
\xi_{k}=x_{k} \text { for } k \leq k_{1}, \quad \xi_{k}=y_{k-k_{1}+k_{2}-1} \text { for } k>k_{1} \text {. }
$$

Suppose that the mild skew product $G$ has OSP. Then there exists a point $q$ such that relation (1.1) is true for it and the pseudotrajectory $\xi$.

By the choice of $\varepsilon$, the corollary to Lemma 1 is valid for the point $r_{1}$ and the pseudotrajectory $\xi$. Hence,

$$
q \in W^{u}\left(r_{1}\right) .
$$


Consider the sequence $\operatorname{pr} \xi$. The points of the sequence $\operatorname{pr} \xi$ coincide with the corresponding points of the trajectory $O\left(\operatorname{pr} r_{2}, \sigma\right)$ before the intersection with the $d / 2$-small neighborhood of the point pr $r_{2}$. Therefore, by relation (1.1), we have

$$
O(\operatorname{pr} x, \sigma) \subset N(\varepsilon, O(\operatorname{pr} q, \sigma)) \text { and } O(\operatorname{pr} q, \sigma) \subset N(\varepsilon, O(\operatorname{pr} x, \sigma))
$$

for sufficiently small $d$. Thus, relation (4.2) and the analog of relation (3.1) are fulfilled for the trajectory of the point $\operatorname{pr} x$. Hence, by the choice of $\varepsilon$ and (4.1),

$$
\operatorname{pr} q \in W^{u}\left(\operatorname{pr} r_{1}\right) \cap W^{s}\left(\operatorname{pr} r_{2}\right) .
$$

All conditions of Lemma 3 are satisfied for the points $\operatorname{pr} x$ and $\operatorname{pr} q$ (of course, we can assume that $\left.\operatorname{dist}\left(x_{1}, q_{1}\right)<\varepsilon\right)$. Hence,

$$
d_{\Sigma^{2}}\left(\sigma^{k}(\operatorname{pr} x), \sigma^{k}(\operatorname{pr} q)\right) \leq R \text { for } k \in \mathbb{Z}
$$

By Lemma 2,

$$
\operatorname{pr} q_{k}=\operatorname{pr} x_{k} \text { for } k \in \mathbb{Z} .
$$

Since $x, q \in W^{u}\left(r_{1}\right)$, we can find a number $K$ such that the points $x_{K}$ and $q_{K}$ belong to a local unstable manifold of the point $r_{1}$ that can be projected to the base injectively (see the choice of $\varepsilon$, item 4)). Hence, the fact that $\operatorname{pr} q_{K}=\operatorname{pr} x_{K}$ implies that $q_{K}=x_{K}$, and the latter yields $q=x$. Consequently, by (1.1), we get the following inclusions:

$$
O(x, G) \subset N(\varepsilon, \xi) \text { and } \xi \subset N(\varepsilon, O(x, G)) \text {, }
$$

which contradicts the construction of the sequence $\xi$. This contradiction means that our assumptions are wrong, and $G \notin \mathrm{OSP}$ in Case (A1).

\section{§5. Start of the proof in Case (A2): Auxiliary lemmas}

Two auxiliary lemmas on properties of skew products required for the proof in Case (A2) will be formulated and verified in this section. We introduce the corresponding notation.

Consider the step skew product $G_{0}$ generated by the diffeomorphisms $g_{0}$ and $g_{1}$. By Theorem 1, $G_{0}$ has infinitely many hyperbolic periodic points of type $(1,2)$ lying on different fibers, and infinitely many hyperbolic periodic points of type $(2,1)$ lying on different fibers. Note that any infinite set of periodic points lying on different fibers in the space $\Sigma^{2} \times S^{1}$ contains points of arbitrarily large periods. Choose four hyperbolic periodic points on different fibers of the step skew product $G_{0}$ : points $p_{1}$ and $p_{3}$ of type $(2,1)$, and points $p_{2}$ and $p_{4}$ of type $(1,2)$.

Recall that any finite sequence of zeros or ones is called a word. The sequence pr $p_{j}$, where $j \in\{1, \ldots, 4\}$, is periodic; i.e., some word $\omega_{j}$ of length $T_{j}$ is repeated periodically in it. We may assume that $\omega_{j}$ is a word of minimal length; i.e., the number $T_{j}$ is the main period of the point $\operatorname{pr} p_{j}$ with respect to the Bernoulli shift $\sigma$. Without loss of generality, we assume that $T_{j}>2$ for $j \in\{1, \ldots, 4\}$; i.e., the word $\omega_{j}$ contains both 0 and 1. Moreover, without loss of generality we assume that

$$
T_{3} \leq \min \left(T_{1}, T_{2}\right) \quad \text { and } \quad T_{4} \leq \min \left(T_{1}, T_{2}\right) .
$$

We introduce the number

$$
T=\max \left(T_{1}, T_{2}\right) .
$$

We can assume that the neighborhoods $W_{0}$ and $W_{1}$ occurring in Theorem $\mathrm{A}^{\prime}$ are so small that the points $p_{j}$ are preserved for any mild skew product (which is, in fact, a perturbation of the step skew product $G_{0}$ ) from Theorem $\mathrm{A}^{\prime}$. This means that the analogs of these points have the same periods and types. In particular, the number $T$ does not depend on the choice of a mild skew product $G$. Let $\delta$ be the maximal radius of the neighborhoods $W_{0}$ and $W_{1}$. We can assume that $\delta$ is an arbitrarily small (and 


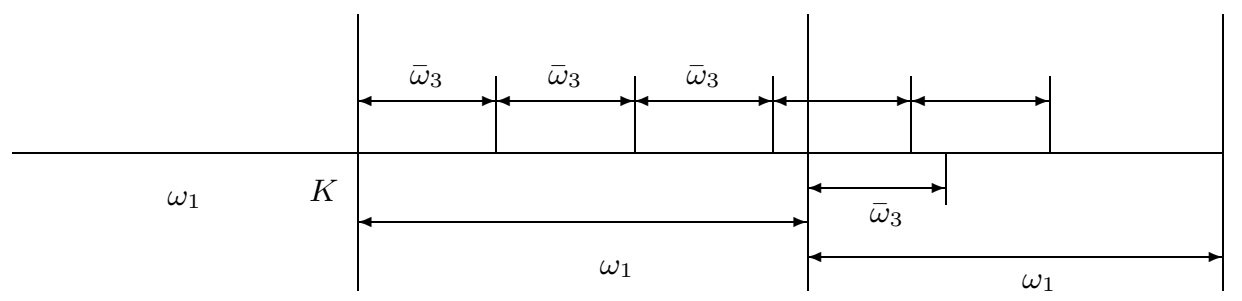

FiguRE 2

dependent on $T$ ) but fixed number. Our main restrictions on the size of $\delta$ will be imposed in $\S 7$.

We denote by the same symbols $p_{j}$ the hyperbolic periodic points of the mild skew product $G$ corresponding to the points $p_{j}$ of the step skew product $G_{0}$. As was noted above, the periods $T_{j}$ and the types of the points $p_{j}$ have not changed. As before, suppose that $\omega_{j}(j \in\{1, \ldots, 4\})$ are periodically repeating words of the sequences $\operatorname{pr} p_{j}$.

We define cylindrical neighborhoods $U_{j}$ of the points $p_{j}(j \in\{1,2\})$ by the formula

$$
U_{j}:=\left\{\omega=\ldots \omega_{j} \omega_{j} \mid \omega_{j} \omega_{j} \ldots\right\} \times S^{1} .
$$

In this formula, the dots denote arbitrary symbols, and the meaning of the sign $\mid$ was explained above (see $\S 2$ ). The word $\omega_{j}$ is repeated four times: two times before the zero position and two times after it. We define cylindrical neighborhoods of the trajectories $O\left(p_{j}, G\right)(j \in\{1,2\})$ by the formula

$$
V_{j}:=U_{j}^{0} \cup U_{j}^{1} \cup \cdots \cup U_{j}^{T_{j}-1},
$$

where the set $U_{j}^{k}\left(0 \leq k \leq T_{j}-1\right)$ is defined like the set $U_{j}$, but the word $\omega_{j}$ is replaced with the word $\sigma^{k}\left(\omega_{j}\right)$, i.e., the corresponding cyclic permutation of the word $\omega_{j}$.

Lemma 4. Under our conditions,

$$
O\left(p_{j}, G\right) \cap V_{t}=\varnothing \quad \text { for } j \in\{3,4\}, t \in\{1,2\} ;
$$

i.e., the trajectories of the points $p_{3}$ and $p_{4}$ do not intersect the cylinder neighborhoods $V_{1}$ and $V_{2}$ of the trajectories $O\left(p_{1}, G\right)$ and $O\left(p_{2}, G\right)$.

Proof. Without loss of generality, we prove relation (5.3) for the point $p_{3}$ and the set $U_{1}$. To get a contradiction, assume that (5.3) fails. This means that there exists a number $K$ such that the word $\omega_{1} \omega_{1} \omega_{1} \omega_{1}$ takes the positions from $K-2 T_{1}$ to $K+2 T_{1}-1$ in the sequence pr $p_{3}$. Also, by (5.1), the word $\omega_{1}$ is longer than $\omega_{3}$. Consider the word $\omega_{1}$ starting from the $K$ th position of the sequence pr $p_{3}$. The first $T_{3}$ symbols of this word are a cyclic permutation of the word $\omega_{3}$. Denote this permutation by $\bar{\omega}_{3}$. Hence, the word $\omega_{1}$ is covered by $m$-times repeated words $\bar{\omega}_{3}$ plus an "addition": the first $r$ symbols of $\bar{\omega}_{3}\left(0 \leq r<T_{3}\right)$.

However, the second word $\omega_{1}$ (which starts from the $\left(K+T_{1}\right)$ th position of the sequence $\operatorname{pr} p_{3}$; see Figure 2) is also covered by the words $\bar{\omega}_{3}$. On the one hand, it should start from $\bar{\omega}_{3}$ (because the first and the second word $\omega_{1}$ coincide); on the other hand, it should start from the last $T_{3}-r$ symbols of $\bar{\omega}_{3}$ (see Figure 2). This means that if we swap the first $r$ symbols and the last $T_{3}-r$ symbols in the word $\bar{\omega}_{3}$, then $\bar{\omega}_{3}$ will not change, i.e., $\sigma^{r}\left(\operatorname{pr} p_{3}\right)=\operatorname{pr} p_{3}$. Hence, $r=0$, but then the word $\omega_{1}$ is the $m$-times repeated word $\bar{\omega}_{3}$. Thus, the trajectories of the points $p_{1}$ and $p_{3}$ intersect, and we get a contradiction with the choice of the points $p_{1}$ and $p_{3}$. Relation (5.3) is proved. 
Lemma 5. Suppose that $m>4 T$ and a sequence $\beta \in \Sigma^{2}$ is such that a word of the form

$$
\omega=\alpha_{1} \omega_{3} \ldots \omega_{3} \alpha_{2}
$$

is repeated in it periodically, where the word $\omega_{3}$ is repeated precisely $m$ times in the formula, and the words $\alpha_{1}$ and $\alpha_{2}$ (whose length is more than 4T) cannot contain less than $T$ zeros in a row (however, it is allowed to contain no zeros at all); then

$$
O(\beta, \sigma) \cap \operatorname{pr} V_{t}=\varnothing \quad \text { for } t \in\{1,2\} .
$$

In other words, the trajectory of the sequence $\beta$ with respect to the Bernoulli shift $\sigma$ that includes the word $\omega$ periodically does not intersect the sets $V_{1}$ and $V_{2}$.

Proof. Without loss of generality, we prove relation (5.4) for the point $\beta$ and the set $\operatorname{pr} U_{1}$. To get a contradiction, suppose the contrary. This means that there exists a number $K$ such that the word $\omega_{1} \omega_{1} \omega_{1} \omega_{1}$ takes the positions from $K-2 T_{1}$ to $K+2 T_{1}-1$ in the sequence $\beta$. Two cases are possible.

a) The $K$ th position is "included" in the word $\alpha_{1}$ (in the case where it is included in the word $\alpha_{2}$, we can apply the same argument). By construction, the word $\omega_{1}$ contains both zeros and ones. Hence, the word $\omega_{1}$ cannot contain more than $T_{1}-1$ zeros. This and the fact that the word $\omega_{1} \omega_{1}$ is contained in the word $\alpha_{1}$ contradict the properties of $\alpha_{1}$.

b) The $K$ th position is "included" in the word $\omega_{3} \ldots \omega_{3}$. Since the word $\omega_{3}$ is repeated $m$ times and $m>4 T$, the word $\omega_{1} \omega_{1}$ is covered by a finite number of words $\omega_{3}$. The further arguments for deriving a 1contradiction are similar to those in the proof of Lemma 4.

We have arrived at a contradiction in both possible cases. Hence, our assumptions are wrong. Lemma 5 is proved.

\section{§6. Reduction of the proof in Case (A2) to Lemma 6}

Recall that a sketch of the proof in Case (A2) was outlined at the beginning of $\S 4$. In this section we give the proof in Case (A2) with the exception of one lemma. Choose a mild skew product $G$ as in Theorem $\mathrm{A}^{\prime}$ and suppose that it satisfies the conditions of Case (A2) (see the beginning of $\S 4$ ). The following lemma plays a key role in the proof of Case (A2). In fact, it states that it is possible to construct pseudotrajectories that are "as precise as we want" and have the required properties (they should "go from" the point $p_{1}$ and "go to" the point $p_{2}$, and their "intermediate part" should be "separated" from the trajectories $O\left(p_{1}, G\right)$ and $\left.O\left(p_{3}, G\right)\right)$.

Lemma 6. Under our conditions, if $\delta$ is sufficiently small, then:

(6.a) the one-dimensional unstable manifold of $p_{1}$ and the two-dimensional stable manifold of $p_{3}$ intersect;

(6.b) the two-dimensional unstable manifold of $p_{4}$ and the one-dimensional stable manifold of $p_{2}$ intersect;

(6.c) given any $d$, there exists a hyperbolic periodic point s such that

- $s \in N\left(d, p_{3}\right)$, and the unstable manifold of $s$ is one-dimensional,

- the trajectory $O(s, G)$ does not intersect the sets $V_{1}$ and $V_{2}$;

(6.d) there exists a point $y \in W^{u}(s) \cap W^{s}\left(p_{4}\right)$ whose trajectory $O(s, G)$ does not intersect $V_{1}$ and $V_{2}$.

Lemma 6 will be proved in $\S 7$. Choose points $x \in W^{u}\left(p_{1}\right) \cap W^{s}\left(p_{3}\right)$ and $z \in W^{u}\left(p_{4}\right) \cap$ $W^{s}\left(p_{2}\right)$. The phase portrait of the mild skew product $G$ is depicted in Figure 3. For convenience, the symbol $s$ denotes all points of the trajectory $O(s, G)$ from Lemma 6 . 


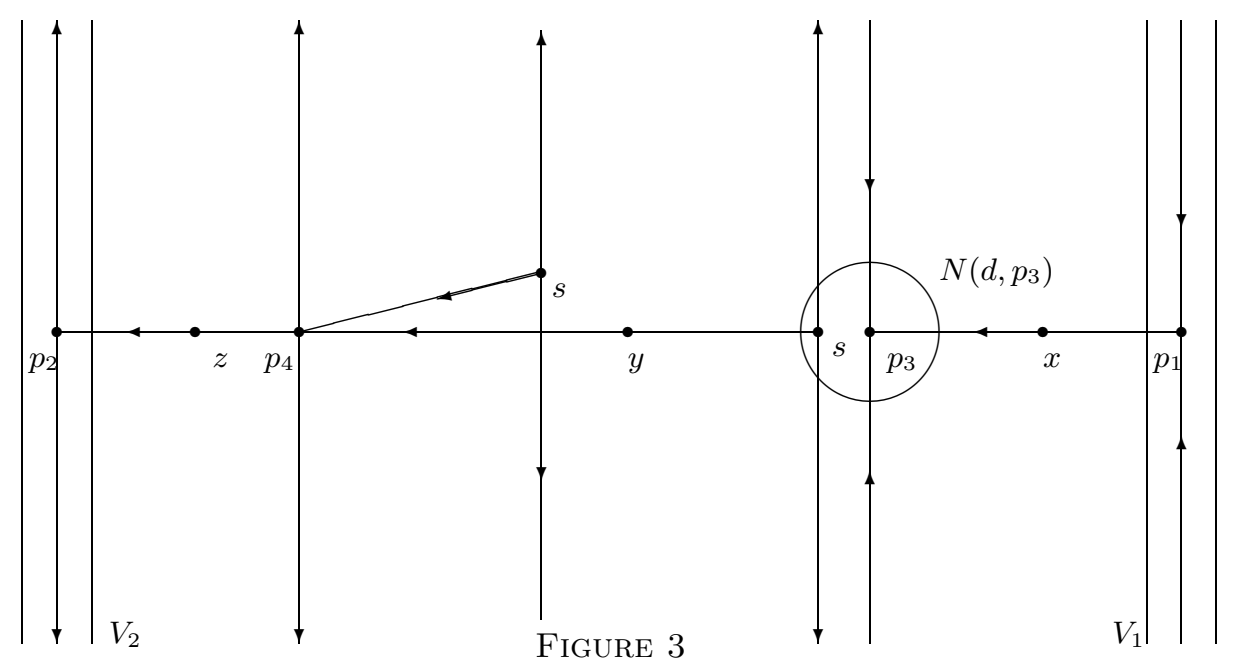

Now we show how to finish the proof for Case (A2) using Lemma 6. First, we need to choose a sufficiently small number $\varepsilon$. We do this in several steps.

The points $x$ and $z$ can be chosen so close to the points $p_{1}$ and $p_{2}$ that there exists a number $\varepsilon_{0}$ such that

$$
\begin{aligned}
& N\left(\varepsilon_{0}, O_{-}(x, G)\right) \subset V_{1}, \quad N\left(\varepsilon_{0}, O_{+}(z, G)\right) \subset V_{2}, \\
& N\left(\varepsilon_{0}, O_{-}(x, G)\right) \cap N\left(\varepsilon_{0}, O(z, g) \cup O(y, G)\right)=\varnothing, \\
& N\left(\varepsilon_{0}, O_{+}(z, G)\right) \cap N\left(\varepsilon_{0}, O(x, G) \cup O(y, G)\right)=\varnothing,
\end{aligned}
$$

where $y$ is an arbitrary point as in Lemma 6 . There exists a number $\varepsilon<\varepsilon_{0} / 3$ such that

- the claim of the Main Lemma holds true for the mapping $G$, the point $z$, and the point $p_{2}$;

- the claim of the corollary to the Main Lemma holds true for the mapping $G$, the point $x$, and the point $p_{1}$.

It was shown in $\S 4$ (see the choice of $\varepsilon_{0}$ ) that both the Main Lemma and its corollary can be applied to the mild skew products.

Now, when $\varepsilon$ is chosen, we can construct the desired pseudotrajectory. Choose an arbitrary number $d<\varepsilon$. Suppose that $s$ is a point corresponding to the number $d / 3$, and $y$ is a point as in item (6.d) corresponding to the point $s$ whose existence was proved in Lemma 6 . There exist numbers $k_{1}, k_{2}, k_{3}$, and $k_{4}$ such that

$$
\begin{array}{ll}
x_{k_{1}+1} \in N\left(d / 3, p_{3}\right), & y_{k_{2}} \in N(d / 3, s), \\
y_{k_{3}+1} \in N\left(d / 2, p_{4}\right), & z_{k_{4}} \in N\left(d / 2, p_{4}\right) .
\end{array}
$$

We construct a $d$-pseudotrajectory $\xi=\left\{\xi_{k}\right\}$ in the following way:

$$
\begin{gathered}
\xi_{k}=x_{k} \text { for } k \leq k_{1}, \quad \xi_{k}=y_{k-k_{1}-1+k_{2}} \text { for } k_{1}<k \leq k_{1}+1+k_{3}-k_{2}, \\
\xi_{k}=z_{k-k_{1}-2-k_{3}+k_{2}+k_{4}} \text { for } k>k_{1}+1+k_{3}-k_{2} .
\end{gathered}
$$

Suppose that the mild skew product $G$ has OSP; i.e., there exists a point $q$ such that relation (1.1) is fulfilled for $q$ and the pseudotrajectory $\xi$.

By the choice of $\varepsilon$, the claim of Lemma 1 is valid for the constructed pseudotrajectory $\xi$ and the point $p_{2}$. Hence,

$$
p \in W^{s}\left(p_{2}\right) .
$$


For similar reasons, the claim of the corollary to Lemma 1 is valid for the pseudotrajectory $\xi$ and the point $p_{1}$. Hence,

$$
p \in W^{u}\left(p_{1}\right) .
$$

But the existence of a point $p$ that satisfies (6.1) and (6.2) contradicts the conditions of Case (A2). This contradiction means that in Case (A2) we have $G \notin$ OSP.

Thus, in both possible cases we have concluded that $G \notin$ OSP. In order to finish the proof of Theorem $\mathrm{A}^{\prime}$, it only remains to prove Lemma 6 .

\section{\$7. Proof of Lemma 6}

The proof of Lemma 6 is based, to a great extent, on the proofs of certain lemmas from [2].

7.1. Item (6.c): main notation. We start from the proof of item (6.c). By Theorem 1 , there exists a hyperbolic periodic point $s$ that satisfies all the conditions of item (6.c) except, perhaps, the last one:

$$
O(s, G) \cap\left(V_{1} \cup V_{2}\right)=\varnothing .
$$

In fact, we repeat the major part of the proof of Theorem 1 (which was formulated in $\S 1)$ in [2, but we need to check that, in addition to other properties, the point $s$ can be required to satisfy relation (7.1). The idea of the proof is to construct the point $s$ so as to satisfy the conditions of Lemma 5 . Then, relation (7.1) holds true by Lemma 5 .

We assume that the sets $W_{0}\left(g_{0}\right)$ and $W_{1}\left(g_{1}\right)$ are balls of radius $\delta$. We introduce the following notation:

$$
\begin{aligned}
\bar{f}_{m}[\omega] & =f_{\sigma^{m-1}(\omega)} \circ \cdots \circ f_{\sigma(\omega)} \circ f_{\omega}, \\
\bar{f}_{-m}[\omega] & =f_{\sigma^{-m}(\omega)}^{-1} \circ \cdots \circ f_{\sigma^{-1}(\omega)}^{-1}, \\
\bar{f}_{0}[\omega] & =\text { id. }
\end{aligned}
$$

Lemma 7 (Lemma on the errors). There exists a number $K$ independent on the choice of $\delta$ such that if

$$
d_{\Sigma^{2}}\left(\omega, \omega^{\prime}\right) \leq 2^{-m}
$$

for a number $m \in N$ and points $\omega, \omega^{\prime} \in \Sigma^{2}$, then

$$
d_{C^{0}}\left(\bar{f}_{ \pm m}[\omega], \bar{f}_{ \pm m}\left[\omega^{\prime}\right]\right) \leq \gamma:=K \delta^{\beta},
$$

where $\beta:=1-\frac{\ln L}{\ln 2^{\alpha}}$, and the numbers $L$ and $\alpha$ are as defined in the conditions of Theorem 1 .

Remark 2. The proof of Lemma 7 is an almost word-for-word repetition of the proof of Lemma 3.1 in 2]: we only need to make some trivial changes concerning the transition to $C^{1}$-topology. We do not give it here.

We need some notation from [2]. Consider the word $\bar{\beta}=\beta_{-m} \ldots \beta_{m-1}$. By definition, put

$$
C_{\bar{\beta}}=\left\{\omega=\left\{\alpha_{k}\right\}_{k \in \mathbb{Z}} \in \Sigma^{2} \mid \alpha_{k}=\beta_{k} \text { for }-m \leq k \leq m-1\right\} .
$$

The set $C_{\bar{\beta}}$ is a cylindrical neighborhood in the set $\Sigma^{2}$. By definition, put

$$
V_{ \pm}[\bar{\beta}](\phi)=\left\{\bar{f}_{ \pm m}[\omega](\phi) \mid \omega \in C_{\bar{\beta}}\right\} .
$$

Put $\Gamma_{m}=C_{\bar{\beta}}$ for a fixed word $\bar{\beta}=\beta_{-m} \ldots \beta_{0} \ldots \beta_{m-1}$ and define the sets $V_{m}(\phi)$ and $V_{-m}(\phi)$ for $\phi \in S^{1}$ by the relations

$$
V_{ \pm m}(\phi)=\left\{\bar{f}_{ \pm m}[\omega](\phi) \mid \omega \in \Gamma_{m}\right\} .
$$

Note that, by definition, $V_{ \pm}[\bar{\beta}](\phi)=V_{ \pm m}(\phi)$. 
By Lemma 7,

$$
\operatorname{diam} V_{ \pm m}(\phi) \leq \gamma,
$$

independently of the choice of a point $\phi$ and the length of $\bar{\beta}$. By the definition of $\gamma$, the number $\delta$ can be chosen in such a way that $\gamma<b / 40$ (the numbers $a$ and $b$ were defined in $\S 2$, when the diffeomorphisms $g_{0}$ and $g_{1}$ were introduced, and these numbers can be thought of as sufficiently close to 1 and 0 , respectively, but fixed in advance).

Note that there exist arcs $W^{+}, W^{-} \subset S^{1}$, whose lengths are at least $1 / 4-\delta$, such that the mapping $f_{\omega}$ expands the arc $W^{+}$(with an expansion constant of at least $a-\delta$ ) and contracts the arc $W^{-}$for any sequence $\omega$ with $\omega_{0}=1$, where the symbol $\omega_{0}$ stands at the zero position. By definition, put

$$
\begin{aligned}
& P=\left\{p \in S^{1} \mid p \text { is the attractor of the mapping } f_{\omega}, \omega \in \Sigma^{2}, \omega_{0}=1\right\}, \\
& Q=\left\{q \in S^{1} \mid q \text { is the repeller of the mapping } f_{\omega}, \omega \in \Sigma^{2}, \omega_{0}=1\right\} .
\end{aligned}
$$

Note that the values of $\operatorname{diam} P$ and $\operatorname{diam} Q$ are of order $\delta$, and they do not exceed $\gamma=K \delta^{\beta}$ if $\delta$ is sufficiently small. Now, when the main notation is introduced, we can formulate and prove the main lemmas.

7.2. Item (6.c): main lemmas. By definition, put $S=[1 /(b-\delta)]$, where [.] denotes the integral part of a number. Note that if $\delta$ is sufficiently small, then $S$ does not depend on the choice of $\delta$.

We need the following lemma, which is a generalization of Lemma 3.3 in [2].

Lemma 8. Let $\alpha=\alpha_{-n} \ldots \alpha_{n-1}$ be a word, and let $\phi_{1}, \phi_{2} \in S^{1}$ be two distinct points. Then there exists a word

$$
\bar{\beta}=\beta_{-m} \ldots \beta_{-n-1} \alpha_{-n} \ldots \alpha_{n-1} \beta_{n} \ldots \beta_{m-1}
$$

such that the words $\beta_{-m} \ldots \beta_{-n-1}$ and $\beta_{n} \ldots \beta_{m-1}$ cannot have less than $T$ zeros in a row and

$$
d_{S^{1}}\left(V_{ \pm}[\bar{\beta}]\left(\phi_{1}\right), V_{ \pm}[\bar{\beta}]\left(\phi_{2}\right)\right) \geq 2 b .
$$

Hereinafter, if no additional remarks are made, we assume that the distance between two sets on the circle is the length of the minimal arc connecting the points of these sets.

Remark 3. In general, the proof of Lemma 8 is similar to the proof of Lemma 3.3 in [2]. All changes in this proof are connected only with the restriction on a number of zeros in the words involved in the lemma. We give only an outline of the proof, making an accent on the necessary changes.

Proof. We construct the word $\beta^{l}=\beta_{-l} \ldots \beta_{l-1}$ inductively, starting with the word $\alpha$ and adding, from one or another end by turns, either $S T+1$ zeros or one unit and $S T$ zeros. We follow the algorithm described below. When the algorithm stops, our construction is completed (we argue by induction on $l$ ). The algorithm consists of two steps.

Step 1. By definition, put

$$
M_{ \pm l}=\min _{\omega \in C_{\beta} l} d_{S^{1}}\left(\bar{f}_{ \pm l}[\omega]\left(\phi_{1}\right), \bar{f}_{ \pm l}[\omega]\left(\phi_{2}\right)\right) .
$$

We check the following conditions:

$$
M_{l}>3 b
$$

and

$$
M_{-l}>3 b
$$


If both conditions are fulfilled, then the algorithm stops; below we prove that in this case the resulting word satisfies our requirements. If at least one of conditions (B1), (B2) is violated, we go to Step 2.

Step 2. By definition, put

$$
W_{ \pm l}=V_{ \pm}\left[\beta^{l}\right]\left(\phi_{1}\right) \cup V_{ \pm}\left[\beta^{l}\right]\left(\phi_{2}\right) .
$$

We check the following conditions:

$$
W_{l} \subset W^{+}
$$

and

$$
W_{-l} \subset W^{-}
$$

If condition (C1) (respectively, condition (C2)) is fulfilled, then we add one unit and $S T$ zeros from the right (respectively, from the left), and if it is violated, we add $S T+1$ zeros likewise. Then we return to Step 1 . Denote by $\mathcal{M}$ the set of all $l$ for which we return to Step 1.

In fact, in [2, Proposition 3.1] it was proved that if the algorithm stops, then the word $\bar{\beta}$ constructed by the algorithm satisfies all the conditions of Lemma 8 .

In order to finish the proof of Lemma 8, it suffices to show that the described algorithm stops after a finite number of steps. Suppose the contrary; i.e., suppose we have constructed some growing sequence of (symmetric) words $\beta^{l}$ that determines a two-sided sequence $\omega$. By definition, put

$$
\begin{aligned}
\phi_{j l}^{ \pm} & =\bar{f}_{ \pm l}[\omega]\left(\phi_{j}\right), \quad j=1,2 ; \\
\delta_{l}^{ \pm} & =d_{S^{1}}\left(\phi_{1 l}^{ \pm}, \phi_{2 l}^{ \pm}\right) .
\end{aligned}
$$

By the construction of the sequence $\omega$, we have $\phi_{j l}^{ \pm} \in V_{ \pm}\left[\beta^{l}\right]\left(\phi_{j}\right)$. Hence, by Lemma 7 and the definition of the numbers $M_{ \pm l}$,

$$
\delta_{l}^{ \pm}-2 K \delta^{\beta} \leq M_{ \pm l} \leq \delta_{l}^{ \pm}
$$

By definition, put

$$
W_{l}^{ \pm}=V_{ \pm}\left[\beta^{l}\right]\left(\phi_{1}\right) \cup V_{ \pm}\left[\beta^{l}\right]\left(\phi_{2}\right) .
$$

Note that $W_{l}^{ \pm}=W_{ \pm l}$. To continue the proof of Lemma 8, we need the following statement.

Proposition 1. There exists a number $m \in \mathcal{M}$ such that $\delta_{m}^{ \pm}>1 / 16$.

Proposition 1 implies that the algorithm described above stops after a finite number of steps. Indeed, the inequality $M_{ \pm m}>3 b$ is true for the number $m \in \mathcal{M}$ from Proposition 1 (of course, if $b$ is sufficiently small compared to $1 / 16$ ).

Proof of Proposition 1. The diffeomorphism $f_{\sigma^{l}(\omega)}$ for $\omega_{l}=0$ maps any point $\phi$ to some point of the arc $[\phi+b-\delta, \phi+b+\delta]$ and maps any arc of length $\lambda$ into an arc of length $\lambda^{\prime} \in((1-\delta) \lambda,(1+\delta) \lambda)$. The diffeomorphism $f_{\sigma^{l}(\omega)}$ for $\omega_{l}=0$ maps any arc of length $\lambda$ that is contained in $W^{+}$into an arc of length $\lambda^{\prime} \in((a-\delta) \lambda,(a+\delta) \lambda)$. Next, if $\omega_{l}=1$, then $\phi_{1 l}^{+}, \phi_{2 l}^{+} \in W^{+}$by construction.

We show that if $\delta_{l}^{+}<1 / 8\left(1 / 8\right.$ is approximately one half of the arc $\left.W^{+}\right)$, then the sequence $\omega$ cannot have more than $(T S+1)(S+1)+T S$ zeros in a row after $\omega_{l}$. Indeed, we apply the mappings $\delta$-close to the rotations through the angles $(T S+1) b$, $(2 T S+2) b, \ldots,(T S+1)(S+1) b$; hence, one of these rotations maps the shortest of the $\operatorname{arcs}\left(\phi_{1 l}^{+}, \phi_{2 l}^{+}\right)$into the arc $W^{+}$(because $W^{+}$is sufficiently large). Suppose that it is the rotation through the angle $(T S+1) \ell b$. If $l-1 \in \mathcal{M}$, then $\omega_{l+(T S+1) \ell b}=1$, by 
construction. Since it is possible that $l-1 \notin \mathcal{M}$, we must take into consideration no more than $S T$ zeros in order to "arrive at" an element of $\mathcal{M}$, i.e., to go to Step 1.

Hence,

$$
\delta_{l+(T S+1)(S+1)+T S}^{+}>(a-\delta)(1-\delta)^{(T S+1)(S+1)+T S} \delta_{l}^{+} \quad \text { for } \quad \delta_{l}^{+}<1 / 8 .
$$

If $\delta$ is sufficiently small, then $a-\delta>1$. Since $(1-\delta)^{(T S+1)(S+1)+T S}$ is close to 1 ,

$$
(a-\delta)(1-\delta)^{(T S+1)(S+1)+T S}>1
$$

if $\delta$ is sufficiently small. Therefore, the sequence $\left\{\delta_{n}^{+}\right\}$contains an element $\delta_{n}^{+} \geq 1 / 8$. We prove that

$$
\delta_{k}^{+}>1 / 16 \text { for all } k>n .
$$

Suppose the contrary; i.e., suppose there exists a number $k>n$ with $\delta_{k}^{+} \leq 1 / 16$. Choose a number $t \in[n, k)$ such that

$$
\delta_{t}^{+} \geq 1 / 8, \quad \delta_{p}^{+}<1 / 8 \text { for all } p \in(t, k] .
$$

Hence, from relation (7.2) it follows that $k<t+(T S+1)(S+1)+T S+1$. Indeed, since $\delta_{t+1}^{+}<1 / 8$, we have

$$
\begin{aligned}
\delta_{t+(T S+1)(S+1)+T S+1}^{+} & >(a-\delta)(1-\delta)^{(T S+1)(S+1)+S T} \delta_{t+1}^{+} \\
& \geq(a-\delta)(1-\delta)^{(T S+1)(S+1)+S T+1} \delta_{t}^{+}>1 / 8 .
\end{aligned}
$$

The last inequality is true because $\delta_{t}^{+} \geq 1 / 8$, and the number $\delta$ can be chosen so small that

$$
(a-\delta)(1-\delta)^{(T S+1)(S+1)+S T+1}>1 .
$$

Thus, $k<t+(T S+1)(S+1)+T S+1$. Similar arguments show that there are no ones among the elements of $\omega_{l}$ for $t+1 \leq l \leq k$. But then

$$
\delta_{k}^{+} \geq(1-\delta)^{(T S+1)(S+1)+2} 1 / 8>1 / 16
$$

(for sufficiently small $\delta$ ), and we get a contradiction with the choice of $k$. This contradiction proves inequality (7.3).

Similarly, it can be proved that there exists a number $n^{\prime}$ such that $\delta_{k}^{-}>1 / 16$ for $k>n^{\prime}$. Our argument implies that we can assume $k, k^{\prime} \in \mathcal{M}$. This completes the proof of Proposition 1.

Therefore, Lemma 8 is proved.

Consider the maximal arcs $\bar{W}^{+}$and $\bar{W}^{-}$such that

$$
N\left(3 \gamma, \bar{W}^{+}\right) \subseteq W^{+} \text {and } N\left(3 \gamma, \bar{W}^{-}\right) \subseteq W^{-} .
$$

Since $\gamma$ is assumed to be sufficiently small, we have

$$
Q \subset \bar{W}^{+} \text {and } P \subset \bar{W}^{-} .
$$

We need the following lemma. It is similar to Lemma 3.4 in 2]: we added only one new item, namely (9.a), and slightly strengthened items (9.b) and (9.c). We give only an outline of the proof of Lemma 9, emphasizing the necessary changes. Also note that Lemma 3.4 in 2] mentioned above involves one more item, which we do not need, so that we omit it. 
Lemma 9 (on distortion of arcs). Suppose that we are given an arc $J \subset S^{1}$ and a word $\bar{\alpha}=\alpha_{-n} \ldots \alpha_{0} \ldots \alpha_{n-1}$. Then there exist words

$$
\bar{\beta}=\beta_{-m} \ldots \beta_{-n-1} \alpha_{-n} \ldots \alpha_{0} \ldots \alpha_{n-1} \beta_{n} \ldots \beta_{m-1}
$$

and

such that

$$
\bar{\beta}^{\prime}=\beta_{-m^{\prime}}^{\prime} \ldots \beta_{-n-1}^{\prime} \alpha_{-n} \ldots \alpha_{0} \ldots \alpha_{n-1} \beta_{n}^{\prime} \ldots \beta_{m^{\prime}-1}^{\prime}
$$

(9.a) the words that were added to $\bar{\alpha}$ cannot contain less than $T$ zeros in a row;

(9.b) if $\omega \in C_{\bar{\beta}}$, then

$$
\begin{aligned}
& \bar{f}_{m}[\omega](J) \subset \bar{W}^{-} \quad \text { and } \quad W^{-} \subset \bar{f}_{-m}[\omega](J), \\
& \left.\left|\left(\bar{f}_{m}[\omega]\right)^{\prime}\right|_{J}|<1, \quad|\left(\bar{f}_{-m}[\omega]\right)^{\prime}\right|_{\left(\bar{f}_{-m}[\omega]\right)^{-1}\left(W^{-}\right)} \mid>1 ;
\end{aligned}
$$

(9.c) if $\omega^{\prime} \in C_{\overline{\beta^{\prime}}}$, then

$$
\begin{aligned}
& \bar{f}_{-m^{\prime}}\left[\omega^{\prime}\right](J) \subset \bar{W}^{+} \quad \text { and } \quad W^{+} \subset \bar{f}_{m^{\prime}}\left[\omega^{\prime}\right](J), \\
& \left.\left|\left(\bar{f}_{-m^{\prime}}\left[\omega^{\prime}\right]\right)^{\prime}\right|_{J}|<1, \quad|\left(\bar{f}_{m^{\prime}}\left[\omega^{\prime}\right]\right)^{\prime}\right|_{\left(\bar{f}_{m^{\prime}}\left[\omega^{\prime}\right]\right)^{-1}\left(W^{+}\right)} \mid>1 .
\end{aligned}
$$

Proof. We construct a word $\bar{\beta}$ with properties (9.a) and (9.b). Denote by $\phi_{1}$ and $\phi_{2}$ the ends of $J$. By Lemma 8, the word $\bar{\alpha}$ can be transformed into a word $\overline{\beta_{1}}=\beta_{-k_{1}} \ldots \beta_{k_{1}-1}$ satisfying the conclusion of Lemma 8; i.e., the distances between the sets $V_{k_{1}}\left(\phi_{1}\right)$ and $V_{k_{1}}\left(\phi_{2}\right)$, and between the sets $V_{-k_{1}}\left(\phi_{1}\right)$ and $V_{-k_{2}}\left(\phi_{2}\right)$ are at least $2 b$.

By definition, put

$$
\begin{aligned}
X_{-l} & :=\bigcap_{\omega \in \Gamma_{l}} \bar{f}_{-l}[\omega](J) & \text { for } l \geq k_{1}, \\
Y_{l} & :=\bigcap_{\omega \in \Gamma_{l}} \bar{f}_{l}[\omega]\left(S^{1}-\bar{J}\right) & \text { for } l \geq k_{1} ;
\end{aligned}
$$

i.e., $X_{-l}$ is the interval between the sets $V_{-l}\left(\phi_{1}\right)$ and $V_{-l}\left(\phi_{2}\right)$ that is contained in the images of the arc $J$ under the mapping $\bar{f}_{-l}[\omega]$, where $\omega$ "runs through" $\Gamma_{l}$, and $Y_{l}$ is the interval between $V_{l}\left(\phi_{1}\right)$ and $V_{l}\left(\phi_{2}\right)$ that is contained in the images of the $\operatorname{arc} S^{1}-\bar{J}$ under the diffeomorphism $\bar{f}_{l}[\omega]$, where $\omega$ "runs through" the set $\Gamma_{l}$ (we denote by $\bar{J}$ the closure of $J$ and by the symbol "-" the set difference).

We transform the word $\bar{\beta}_{1}$ into the word $\bar{\beta}_{2}=\beta_{-k_{2}} \ldots \beta_{k_{2}-1}$ in such a way that $\bar{\beta}_{2}$ satisfies item (9.a) and the inclusions

$$
Q \subset Y_{k_{2}} \text { and } P \subset X_{-k_{2}} .
$$

For this, we use induction (as in Lemma 8) to add symbols both from the left and from the right. Let $l$ be the induction parameter, the case $l=k_{1}$ being the induction base.

We check the following two conditions:

$$
Q \subset Y_{l}
$$

and

$$
P \subset X_{-l} .
$$

If both conditions are fulfilled, the construction is completed. Otherwise, we do the following: if condition (D1) (respectively, (D2)) is satisfied, then we add $S T+1$ ones from the right (from the left); otherwise we add $S T+1$ zeros in the same maner.

Now we show that this algorithm stops after a finite number of iterations.

Proposition 2. If either condition (D1) or condition (D2) is fulfilled at some iteration, then it will be fulfilled up to the end of the construction. 
The essence of this proposition can be formulated as follows: addition of any number of ones cannot "hurt" these conditions. The proof of Proposition 2 (as well as its formulation) is a word-for-word repetition of the proof of Proposition 3.4 in [2], and we omit it.

Proposition 3. Each of conditions (D1) and (D2) is satisfied at some iteration.

Proof of Proposition 3. Suppose that condition (D1) is never fulfilled; the case of condition (D2) can be treated in the same way. The algorithm described above determines a certain sequence $\omega$, and, by Proposition $2, \omega_{l}=0$ for $l \geq k_{1}$. Hence, the mappings $f_{\sigma^{l}(\omega)}$ are close to rotation by the angle $b$ for $l \geq k_{1}$. Moreover, by the choice of $k_{1}$, the distance between the sets $V_{k_{1}}\left(\phi_{1}\right)$ and $V_{k_{1}}\left(\phi_{2}\right)$ is $2 b$ at least. Hence,

$$
\operatorname{diam}\left(\bar{f}_{k_{1}}[\omega]\left(S^{1}-\bar{J}\right)\right) \geq 2 b .
$$

But then, if $\delta$ is sufficiently small, one of the arcs

$$
\bar{f}_{k_{1}}[\omega]\left(S^{1}-\bar{J}\right), \bar{f}_{k_{1}+S T+1}[\omega]\left(S^{1}-\bar{J}\right), \ldots, \bar{f}_{k_{1}+(S+1) S T+S+1}[\omega]\left(S^{1}-\bar{J}\right)
$$

covers $Q$ in such a way that the distance from $Q$ to the ends of that arc exceeds $\gamma$. Let it be the arc $\bar{f}_{k_{1}+t S T+t}[\omega]\left(S^{1}-\bar{J}\right)$. By Lemma 7 , the sets $V_{k_{1}+t S T+t}\left(\phi_{1}\right)$ and $V_{k_{1}+t S T+t}\left(\phi_{2}\right)$ are contained in the $\gamma$-neighborhoods of the ends of this arc. Therefore, we have $Q \subset$ $Y_{k_{1}+t S T+t}$, and condition (D1) is satisfied.

Thus, we have constructed a word $\bar{\beta}_{2}$ of length $2 k_{2}$ that satisfies the analogs of condition (9.a) from the formulation of the lemma and conditions (D1) and (D2). To finish the proof, it remains to repeat the argument from the end of the proof of Lemma 3.4 in [2] with minor changes. Lemma 9 is proved.

Now, when Lemma 9 is proved, we can finish the proof of item (6.c).

7.3. Item (6.c): end of the proof. Note that sets of the form $C_{\alpha} \times J \subset \Sigma^{2} \times S^{1}$, where $J \subset S^{1}$ is an arc and $\alpha$ is a word, form a base of topology in the set $\Sigma^{2} \times S^{1}$. As above, suppose that $\omega_{3}$ is a word repeating periodically (with period $T_{3}$ ) in the sequence $\operatorname{pr} p_{3}$. We choose a sufficiently large number $2 m$ and a small enough arc $J$ that

$$
p_{3} \subset C_{\omega_{3} \ldots \omega_{3}} \times J \subset N\left(d, p_{3}\right),
$$

where the word $\omega_{3}$ is repeated precisely $m$ times before the zero position and precisely $m$ times after. Next, we apply item (9.c) of Lemma 9. Suppose that $\bar{\beta}$ is the word in item (9.c) of Lemma 9. Let $\omega$ denote an infinite sequence in which the word $\bar{\beta}$ is repeated periodically (and $\omega \in C_{\bar{\beta}}$ ). For any point $\phi \in S^{1}$, Lemma 5 applies to the point $(\omega, \phi) \in \Sigma^{2} \times S^{1}$ (because property (9.a) holds). Hence, condition (7.1) is satisfied for any point $s=(\omega, \phi)$.

In [2, it was proved that there exists a point $\phi_{0} \in S^{1}$ such that the point $s:=\left(\omega, \phi_{0}\right)$ is a hyperbolic periodic point of type $(1,2)$, and conditions $(7.6)$ and $(7.7)$ are fulfilled. Item (6.c) is proved.

7.4. Proof of the remaining items of Lemma 6. We only give the proof of item (6.d). Note that the points $p_{1}, p_{2}, p_{3}$, and $p_{4}$ are preserved for the mild skew products $G$ under consideration, and there was a lot of freedom in the choice of these points (indeed, only hyperbolicity and condition (5.1) on periods were required). Subsections 7.1-7.3 contain a fairly detailed exposition of the procedure described in [2] for constructing hyperbolic periodic points $p=(\omega, \phi)$ of different types that satisfy conditions $(7.4)$ and (7.5), or (7.6) and (7.7), respectively (depending on the type of the periodic point). This procedure allows us to construct points of arbitrarily large periods. Therefore, it can be assumed that the points $p_{1}$ and $p_{4}$ were initially constructed by such a procedure for the 
step skew product $G_{0}$ and then fixed. In this case, items (6.a) and (6.b) are consequences of item (6.d). Thus, it suffices to prove only item (6.d).

We give a brief outline of the method of proof of (6.d). First, we construct a pseudotrajectory $\omega \in \Sigma^{2}$ such that

$$
\omega \in W^{u}(\operatorname{pr} s) \cap W^{s}\left(\operatorname{pr} p_{4}\right) \quad \text { and } \quad O(\omega, \sigma) \cap\left(\operatorname{pr} V_{1} \cup \operatorname{pr} V_{2}\right)=\varnothing ;
$$

the sequence $\omega$ "includes" a certain subsequence of the sequence pr $s$ before the zero position, "includes" zeros from the zero position to the $(\bar{K}-1)$ st position and "includes" a certain subsequence of the sequence pr $p_{4}$ after the $\bar{K}$-position (the number $\bar{K}$ is arbitrary at the moment; later it will be chosen implicitly). Then, we prove that for the sequence $\omega$ there exists a point $\phi \in S^{1}$ such that the trajectory of the point $(\omega, \phi)$ "goes" from the point $s$ to the point $p_{4}$. Next, we apply Proposition 5 (an analog of Lemma 5 ) to see that the trajectory of the point $y:=(\omega, \phi)$ does not intersect the cylindrical neighborhoods $V_{1}$ and $V_{2}$ of the sets $O\left(p_{1}, G\right)$ and $O\left(p_{2}, G\right)$. Thus, the claim of item (6.d) holds true for $y$.

Choose arbitrary numbers $\bar{K} \in \mathbb{Z}$ and $m \in \mathbf{N}$. We say that two sequences $\omega=\left\{\beta_{k}\right\}_{k \in \mathbb{Z}}$ and $\bar{\omega}=\left\{\bar{\beta}_{k}\right\}_{k \in \mathbb{Z}}$ coincide on the interval $[\bar{K}-m, \bar{K}+m-1]$ if

$$
\beta_{k}=\bar{\beta}_{k} \text { for } \bar{K}-m \leq k \leq \bar{K}+m-1 .
$$

We need the following statement; formally, it is a generalization of Lemma 7 on errors. Its proof is trivial.

Proposition 4. If sequences $\omega, \bar{\omega} \in \Sigma^{2}$ satisfy (7.8), then

$$
d_{S^{1}}\left(\bar{f}_{\bar{K} \pm m}[\omega]\left(\bar{\phi}_{1}\right), \bar{f}_{\bar{K} \pm m}[\bar{\omega}]\left(\bar{\phi}_{2}\right)\right) \leq \gamma,
$$

where $\gamma$ is the constant from Lemma 7 , and $\bar{\phi}_{1}, \bar{\phi}_{2} \in S^{1}$ are the points defined by the formulas

$$
\bar{\phi}_{1}:=\left(\bar{f}_{\bar{K}}[\omega]\right)^{-1}(\phi) \in S^{1}, \quad \bar{\phi}_{2}:=\left(\bar{f}_{\bar{K}}[\bar{\omega}]\right)^{-1}(\phi) \in S^{1} .
$$

Recall that the number $T$ is defined by (5.2). Denote by $t_{p}$ the period of the point $p_{4}$ and by $t_{s}$ the period of $s$. Note that $t_{s}>T$. We may assume that $p_{4}=\left(\alpha^{p}, \phi_{p}\right)$, and a word $\alpha_{1}^{p} \ldots \alpha_{t_{p}}^{p}$ is repeated periodically in the sequence $\alpha^{p}$ in such a way that the symbol $\alpha_{1}^{p}$ stands at the zero position of the sequence $\alpha^{p}$. Recall that the points $s$ and $p_{4}$ are repellers on the fiber. In Subsection 6.3, we chose a maximal $\operatorname{arc} W^{+}$such that

$$
N\left(3 \gamma, \bar{W}^{+}\right) \subset W^{+} .
$$

Since the point $p_{4}=\left(\alpha^{p}, \phi_{p}\right)$ is periodic, we have

$$
\bar{f}_{t_{p}}\left[\alpha^{p}\right]\left(\phi_{p}\right)=\phi_{p} .
$$

The construction of the point $s$ (by item (9.c) of Lemma 9) implies that analogs of relations (7.6) and (7.7) hold true for the sequence $\omega^{s}=\operatorname{pr} s$, the arc $J$, and a certain number $m_{s}$ (defined in Lemma 9). Hence, if $\bar{s}=\left(\alpha^{s}, \phi_{s}\right)=G^{m_{s}}(s)$, and a word $\alpha_{1}^{s} \ldots \alpha_{t_{s}}^{s}$ is repeated periodically in the sequence $\alpha^{s}$ in such a way that the symbol $\alpha_{t_{s}}^{s}$ stands at the $(-1)$ st position of the sequence $\alpha^{s}$, then

$$
\phi_{s} \subset \bar{W}^{+} \subset W^{+}, \quad \bar{f}_{-t_{s}}\left[\alpha^{s}\right]\left(W^{+}\right) \subset \bar{W}^{+} \subset W^{+}, \quad\left|\left(\bar{f}_{-t_{s}}\left[\alpha^{s}\right]\right)^{\prime}\right|_{W^{+}} \mid<1 .
$$

These relations mean that the $\operatorname{arc}\left(\alpha^{s}, W^{+}\right)$is contained in the repelling domain of the point $\bar{s}$ with respect to the mapping $G^{t_{s}}$; i.e., the repelling domain on the fiber of the point $\left(\alpha^{s}, \phi_{s}\right)$ (the repelling domain of the point $\phi_{s}$ for the restriction of the mapping $G^{t_{s}}$ to the set $\left.\left(\alpha^{s}, S^{1}\right)\right)$ contains the $\operatorname{arc} W^{+}$.

Since the point $\bar{s}=\left(\alpha^{s}, \phi_{s}\right)$ is periodic, we have

$$
\bar{f}_{-t_{s}}\left[\alpha^{s}\right]\left(\phi_{s}\right)=\phi_{s} .
$$


Choose a neighborhood $\Delta_{p}$ of $p_{4}$ such that if

$$
O_{+}\left(p, G^{t_{p}}\right) \subset \Delta_{p}
$$

for some point $p$, then $p \in W^{s}\left(p_{4}\right)$.

Assume that the sequence $\omega$ is such that

(1) it includes a word $\alpha_{1}^{s} \ldots \alpha_{t_{s}}^{s}$ from the $\left(-t_{s}\right)$ th position to the $(-1)$ st position, and this word is further periodically repeated in the subsequence $\omega_{k<0}$ (the sequence $\omega$ can be viewed as the mapping $\omega: \mathbb{Z} \mapsto M$; then $\left.\omega\right|_{A}$ is the restriction of $\omega$ to a set $A)$;

(2) it includes a word $\alpha_{1}^{p} \ldots \alpha_{t_{p}}^{p}$ from the $\bar{K}$ th position to the $\left(\bar{K}+t_{p}-1\right)$ st position, and this word is further periodically repeated in the subsequence $\left.\omega\right|_{k \geq \bar{K}}$, where $\bar{K} \in \mathbf{N}$ is a certain number to be chosen later.

Lemma 10. (10.a) For any number $m \in \boldsymbol{N}$, there exists a point $\phi_{\omega}^{m}$ and an arc $J_{\omega}^{m}$ such that

$$
\begin{aligned}
& \phi_{\omega}^{m} \in N\left(\gamma, \phi_{p}\right) ; \\
& \bar{W}^{+} \subset N\left(\gamma, J_{\omega}^{m}\right) \quad \text { and } \quad J_{\omega}^{m} \subset N\left(\gamma, \bar{W}^{+}\right) \subset W^{+} ; \\
& d_{S^{1}}\left(\bar{f}_{w t_{p}}\left[\sigma^{\bar{K}}(\omega)\right]\left(\phi_{\omega}^{m}\right), \phi_{p}\right) \leq \gamma \quad \text { for } \quad 0 \leq w \leq 2 m ; \\
& \bar{f}_{-w t_{s}}[\omega]\left(J_{\omega}^{m}\right) \subset N\left(\gamma, \bar{f}_{-w t_{s}}\left[\alpha^{s}\right]\left(\bar{W}^{+}\right)\right) \subset W^{+} \quad \text { for } \quad 0 \leq w \leq 2 m .
\end{aligned}
$$

(10.b) If $\phi_{\omega}$ is a limit point of the sequence $\phi_{\omega}^{m}$, and an arc $J_{\omega}$ is a "limit arc" of the sequence $J_{\omega}^{m}$ (the meaning of this term will be clarified in the proof of the lemma), then

$$
\left(\sigma^{\bar{K}}(\omega), \phi_{\omega}\right) \in W^{s}\left(p_{4}\right), \quad\left(\omega, J_{\omega}\right) \subset W^{u}(\bar{s}) .
$$

Proof. We start with the proof of item (10.a). Choose an arbitrary number $m \in \mathbf{N}$ and an arbitrary number $0 \leq k<m$.

We put $\bar{L}=m t_{p}$ and consider the sequence $\alpha^{p}$. Next, we apply Proposition 4 to the "interval" $\left[\bar{L}-(m-k) t_{p}, \bar{L}+(m-k) t_{p}-1\right]=\left[k t_{p}, 2 m t_{p}-k t_{p}-1\right]$ and the sequences $\sigma^{\bar{K}}(\omega)$ and $\alpha^{p}$, which coincide on this interval by construction. Put $\phi_{\omega}^{m}=\left(\bar{f}_{m t_{p}}\left[\sigma^{\bar{K}}(\omega)\right]\right)^{-1}\left(\phi_{p}\right)$. By construction,

$$
\bar{f}_{t_{p}}\left[\alpha^{p}\right]\left(\phi_{p}\right)=\phi_{p} .
$$

Hence, by inequalities (7.9) and (7.10), we have

$$
\begin{aligned}
& d_{S^{1}}\left(\bar{f}_{k t_{p}}\left[\sigma^{\bar{K}}(\omega)\right]\left(\phi_{\omega}^{m}\right), \bar{f}_{k t_{p}}\left[\alpha^{p}\right]\left(\phi_{p}\right)\right) \leq \gamma \\
& d_{S^{1}}\left(\bar{f}_{2 m t_{p}-k t_{p}}\left[\sigma^{\bar{K}}(\omega)\right]\left(\phi_{\omega}^{m}\right), \bar{f}_{2 m t_{p}-k t_{p}}\left[\alpha^{p}\right]\left(\phi_{p}\right)\right) \leq \gamma
\end{aligned}
$$

for any $0 \leq k<m$. Next, we set $k=0$ in inequality (7.16), obtaining (7.11). Inequalities (7.13) for $w \neq m$ follow from (7.16) and (7.17). Inequalities (7.13) for $w=m$ are true by construction.

Put $\bar{L}=-m t_{s}$. We apply Proposition 4 to the "interval"

$$
\left[\bar{L}-(m-k) t_{s}, \bar{L}+(m-k) t_{s}-1\right]=\left[-2 m t_{s}+k t_{s},-k t_{s}-1\right]
$$

and the sequences $\omega$ and $\alpha^{s}$, which coincide on this interval by construction. Put $V_{k}^{+}=\bar{f}_{-k t_{s}}\left[\alpha^{s}\right]\left(\bar{W}^{+}\right)$and $J_{\omega}^{m}=\left(\bar{f}_{-m t_{s}}[\omega]\right)^{-1}\left(V_{m}^{+}\right)$. Clearly, the set $J_{\omega}^{m}$ is an arc. By construction, the set $\left(\alpha^{s}, W^{+}\right)$is contained in the repelling domain of the point $\bar{s}$, and moreover,

$$
\bar{f}_{v t_{s}}\left[\alpha^{s}\right]\left(W^{+}\right) \subset \bar{W}^{+} \subset W^{+} \quad \text { for } v \in \mathbb{Z}, v \leq 0 .
$$

By the construction of the arc $\bar{W}^{+}$,

$$
V_{-v}^{+}=\bar{f}_{v t_{s}}\left[\alpha^{s}\right]\left(\bar{W}^{+}\right) \subset \bar{W}^{+} \quad \text { for } \quad v \in \mathbb{Z}, v \leq 0 .
$$


Hence, by (7.9) and (7.10),

$$
\begin{aligned}
& d_{H}\left(\bar{f}_{-2 m t_{s}+k t_{s}}[\omega]\left(J_{\omega}^{m}\right), V_{2 m-k}^{+}\right) \leq \gamma, \\
& d_{H}\left(\bar{f}_{-k t_{s}}[\omega]\left(J_{\omega}^{m}\right), V_{k}^{+}\right) \leq \gamma
\end{aligned}
$$

for all $0 \leq k<m$, where $d_{H}$ denotes the Hausdorff distance.

Next, we set $k=0$ in (7.19), obtaining (7.12). Inequalities (7.14) for $w \neq m$ follow from (7.18) and (7.19). Inequalities (7.14) for $w=m$ are true by construction.

We prove item (10.b). Let $\phi_{\omega}$ be a limit point of the sequence $\phi_{\omega}^{m}$. Then, relations (7.13) and (7.11) are valid for the point $\phi_{\omega}$ and an arbitrary number $w$. Let $j_{1}^{m}$ and $j_{2}^{m}$ be the ends of the arc $J_{\omega}^{m} \subset \bar{W}^{+}$. Then there exists a sequence $m_{k}$ such that

$$
j_{e}^{m_{k}} \longrightarrow j_{e} \text { for } e=\{0,1\}, k \rightarrow+\infty,
$$

where $j_{1}$ and $j_{2}$ are some points. Let $J_{\omega}$ be the arc between $j_{1}$ and $j_{2}$ contained in $W^{+}$. The arc $J_{\omega}$ is called the limit arc. Note that $J_{\omega}$ is the set of all limit points of the sequence $J_{\omega}^{m_{k}}$. The arc $J_{\omega}$ satisfies relations (7.14) and (7.12) for arbitrary $w$.

Put

$$
\bar{\Delta}_{p}=\left\{p_{4}+\left(x-p_{4}\right) / 2 \mid x \in \Delta_{p}\right\} .
$$

Since the points $p_{1}, p_{2}, p_{3}$, and $p_{4}$ were "fixed" for all mild skew products from Theorem $\mathrm{A}^{\prime}$, we can assume that $\delta$ was chosen to be so small that

$$
N\left(2 \gamma, \bar{\Delta}_{p}\right) \subset \Delta_{p}
$$

By (7.13), the positive semitrajectory of the point $\left(\sigma^{\bar{K}}(\omega), \phi_{\omega}\right)$ with respect to the mapping $G^{t_{p}}$ is contained in the neighborhood $\bar{\Delta}_{p}$ (for sufficiently small $\gamma$; recall that the point $p_{4}$ is fixed, and the point $\bar{s}$ is not fixed). Consequently,

$$
\left(\sigma^{\bar{K}}(\omega), \phi_{\omega}\right) \in W_{G^{t p}}^{s}\left(p_{4}\right) \text {, whence }\left(\sigma^{\bar{K}}(\omega), \phi_{\omega}\right) \in W^{s}\left(p_{4}\right) .
$$

Similarly, by (7.14), the negative semitrajectories of the points of the arc $\left(\omega, J_{\omega}\right)$ belong to a small neighborhood of the $\operatorname{arc}\left(\operatorname{pr} \bar{s}, \bar{W}^{+}\right)$.

It has already been noted that the repelling domain of the point $\bar{s}$ on the fiber with respect to the mapping $G^{t_{s}}$ contains the arc $W^{+}$, and therefore, the repelling domain of the point $\bar{s}$ with respect to the mapping $G^{t_{s}}$ is sufficiently large; specifically, it contains a subset of the form

$$
\left(V(\operatorname{pr} \bar{s}) \cap W_{\sigma}^{u}(\operatorname{pr} \bar{s}), N\left(\gamma, \bar{W}^{+}\right)\right),
$$

where $V(\operatorname{pr} \bar{s})$ is a small neighborhood of the point $\operatorname{pr} \bar{s}$ in the base, i.e., in the set $\Sigma^{2}$.

Thus, relations (7.14) imply the inclusion

$$
\left(\omega, J_{\omega}\right) \subset W_{G^{t_{s}}}^{u}(\bar{s}), \text { whence }\left(\omega, J_{\omega}\right) \subset W^{u}(\bar{s}) .
$$

Lemma 10 is proved.

In particular, Lemma 10 means that relations (7.15) hold true for a certain point $\phi_{\omega}$ and a certain $\operatorname{arc} J_{\omega}$. We determine the symbols contained in the "interval" from the zero position to the $(\bar{K}-1)$ st position in the sequence $\omega$. Recall that in Subsection 7.2 we introduced the number $S=[1 /(b-\delta)]$, where number $b$ is such that the diffeomorphism $g_{0}$ defined above is the rotation through the angle $b$. By (7.12), the arc $J_{\omega}$ is sufficiently large; consequently, one of the arcs

$$
\bar{f}_{T S+1}[\omega]\left(J_{\omega}\right), \ldots, \bar{f}_{k T S+k}[\omega]\left(J_{\omega}\right), \ldots, \bar{f}_{(S+1) T S+S+1}[\omega]\left(J_{\omega}\right)
$$

"covers" the point $\phi_{p}$. Suppose that this happens for the arc $\bar{f}_{k T S+k}[\omega]\left(J_{\omega}\right)$. In this case, by definition, we put

$$
\bar{K}:=k T S+k,
$$


and define all symbols of the sequence $\omega$ in the interval from the zero position to the $(\bar{K}-1)$ st position to be equal to zero.

By Lemma 10, $\left(\omega, J_{\omega}\right) \subset W^{u}(\bar{s})$ and $\left(\sigma^{\bar{K}}(\omega), \phi_{p}\right) \in W^{s}\left(p_{4}\right)$. By construction, we have $\left(\sigma^{\bar{K}}(\omega), \phi_{p}\right) \in G^{\bar{K}}\left(\omega, J_{\omega}\right) ;$ consequently,

$$
\left(\sigma^{\bar{K}}(\omega), \phi_{p}\right) \in W^{u}(\bar{s}) \cap W^{s}\left(p_{4}\right) .
$$

In order to finish the proof of Lemma 6 , we only need to prove the following statement. Since the proof of it is similar to that of Lemma 5 , we omit it.

Proposition 5. Suppose that a word $\beta_{p}$ is repeated periodically with period $T_{4}$ in the sequence $\operatorname{pr} p_{4}$. Let $\beta_{s}=\alpha_{1}^{s} \ldots \alpha_{t_{s}}^{s}$ be the word (constructed above) that is periodically repeated in the sequence $\operatorname{pr} s$, and let $\theta$ be a word that consists of $k(T S+1)$ zeros for $k \geq 0$. If $\omega=\ldots \beta_{s} \ldots \beta_{s} \theta \beta_{p} \ldots \beta_{p} \ldots$, then

$$
O(\omega, \sigma) \cap\left(\operatorname{pr} V_{1} \cup \operatorname{pr} V_{2}\right)=\varnothing .
$$

Now, it remains to apply Proposition 5 to the point $y:=\left(\sigma^{\bar{K}}(\omega), \phi_{p}\right)$ to see that the point $y$ satisfies all conditions of item (6.d). Lemma 6 is proved. Hence, Theorems A and $\mathrm{A}^{\prime}$ are also proved.

\section{REFERENCES}

[1] A. S. Gorodetskiu and Yu. S. Ilyashenko, Some new robust properties of invariant sets and attractors of dynamical systems, Funktsional. Anal. i Prilozhen. 33 (1999), no. 2, 16-30; English transl., Funct. Anal. Appl. 33 (1999), no. 2, 95-105. MR1719330 (2001c:37031)

[2] _ Some properties of skew products over a horseshoe and a solenoid, Tr. Mat. Inst. Steklov. 231 (2000), 96-118; English transl., Proc. Steklov Inst. Math. 2000, no. 4 (231), 90112. MR 1841753 (2002i:37040)

[3] A. S. Gorodetskiǔ, Minimal attractors and partially hyperbolic invariant sets of dynamical systems, Diss., Moskov. Gos. Univ., Moscow, 2001. (Russian)

[4] V. A. Kleptsyn and M. B. Nal'skiŭ, Stability of the existence of nonhyperbolic measures for $C^{1}$-diffeomorphisms, Funktsional. Anal. i Prilozhen. 41 (2007), no. 4, 30-45; English transl., Funct. Anal. Appl. 41 (2007), no. 4, 271-283. MR2411604 (2009k:37047)

[5] S. Yu. Pilyugin, Spaces of dynamical systems, Inst. Kompyuter. Issled., Moscow-Izhevsk, 2008. (Russian)

[6] Ch. Bonatti, L. J. Diaz, and G. Turcat, Pas de "shadowing lemma" pour des dynamiques partiellement hyperboliques, C. R. Acad. Sci. Paris Sér. I Math. 330 (2000), 587-592. MR1760444 (2001d:37033)

[7] S. Crovisier, Periodic orbits and chain-transitive sets of $C^{1}$-diffeomorphisms, Publ. Math. Inst. Hautes Études Sci. No. 104 (2006), 87-141. MR2264835 (2008f:37047)

[8] A. S. Gorodetskiū, Regularity of central leaves of partially hyperbolic sets and applications, Izv. Ross. Akad. Nauk Ser. Mat. 70 (2006), no. 6, 19-44; English transl., Izv. Math. 70 (2006), no. 6, 1093-1116. MR2285025 (2007k:37033)

[9] M. W. Hirsch, C. C. Pugh, and M. Shub, Invariant manifolds, Lecture Notes in Math., vol. 583, Springer-Verlag, Berlin-New York, 1977. MR0501173 (58:18595)

[10] A. Katok and B. Hasselblatt, Introduction to the modern theory of dynamical systems, Encyclopedia Math. Appl., vol. 54, Cambridge Univ. Press, Cambridge, 1995. MR.1326374 (96c:58055)

[11] K. Palmer, Shadowing in dynamical systems. Theory and applications, Math. Appl., vol. 501, Kluwer Acad. Publ., Dordrecht, 2000. MR.1885537 (2002k:37034)

[12] S. Yu. Pilyugin, A. A. Rodionova, and K. Sakai, Orbital and weak shadowing properties, Discrete Contin. Dyn. Syst. 9 (2003), 287-308. MR.1952375 (2003j:37039)

[13] S. Yu. Pilyugin, Shadowing in dynamical systems, Lecture Notes in Math., vol. 1706, SpringerVerlag, Berlin, 1999. MR1727170 (2001b:37030) 
A. V. OSIPOV

[14] S. Yu. Pilyugin, K. Sakai, and O. A. Tarakanov, Transversality properties and $C^{1}$-open sets of diffeomorphisms with weak shadowing, Discrete Contin. Dyn. Syst. 16 (2006), no. 4, 871-882. MR2257163(2008g:37019)

[15] S. Yu. Pilyugin and O. B. Plamenevskaya, Shadowing is generic, Topology Appl. 97 (1999), 253266. MR1711347 (2000i:37023)

Mathematics and Mechanics Department, St. Petersburg State University, 28 UniversitetSkiI Prospekt, Stary Peterhof, St. Petersburg 198504, Russia

E-mail address: osipovav@list.ru

Received 28/FEB/2009

Translated by THE AUTHOR 